\title{
THE SPECTRAL FEATURES OF POLYATOMIC MOLECULES MULTIPHOTON EXCITATION IN A STRONG IR-LASER FIELD
}

\author{
S. S. ALIMPIEV and B. G. SARTAKOV \\ General Physics Institute, Vavilov st. 38, Moscow, 117942, Russia
}

The experimental data on multiphoton excitation of polyatomic molecules in strong fields of high pressure continuously tunable $\mathrm{CO}_{2}$ lasers are presented. The program package for computer simulation of multiphoton excitation spectra and excitation dynamics is developed. Good agreement between computer simulation and experimental data is observed.

\section{INTRODUCTION}

Since the first observation of multiphoton dissociation (MPD) of polyatomic molecules in a strong IR laser field ${ }^{1}$ and demonstration of isotopic selectivity of $\mathrm{MPD}^{2}$ the process of collisionless multiphoton excitation (MPE) of polyatomic molecules have been investigated in many laboratories (see reviews ${ }^{3-4}$ ).

It was demonstrated that the main features of MPE are dominated by the complex structure of vibrational-rotational spectrum of polyatomic molecules. In particular, it was generally accepted to divide vibrational spectrum of polyatomic molecules into two qualitatively different regions: the region of low lying discrete levels (DL) and the region called quasicontinuum (QC) of vibrational states. Respectively MPE has qualitatively different features in these two regions. MPE in the DL region is the coherent excitation via one and multiphoton transitions. The efficiency of excitation in this region reveals the sharp resonance dependence on laser frequency that is determined by the spectral structure of rotation-vibration spectrum of DL. It is this stage of excitation that provides the isotopic selectivity of MPD. In contrast, the incoherent excitation in QC via ladder switching one photon transitions is the dominating process of MPE because the anharmonicity mode mixing leads to the high density of one photon dipole allowed transitions. The form factor of such one photon bands in QC is assumed to be a widely broadened. It explains the smooth dependence of the MPE efficiency in QC region on the excitation frequency. The ladder switching excitation in QC provides the high efficiency of MPD at moderate intensity of exciting radiation with fixed frequency. The qualitative difference of the excitation process in first and second stages makes it possible to search the optimal way of MPD by using of two or three laser frequency irradiation with each laser pulse frequency being optimal for an appropriate excitation stage. The multifrequency 
excitation of polyatomic molecules gives reach opportunities to conserve the sharp resonance structure and to dissociate a molecule with high efficiency simultaneously. To realize these opportunities as the experimental investigation of spectral features of multifrequency excitation as soon as the theoretical analysis of excitation process based on the comprehensive set of molecular spectroscopic constants are necessary.

The subject of this work is the survey analysis of experimental data on MPE, MPD spectroscopy obtained in the last years in our group with the help of continuously tunable $\mathrm{CO}_{2}$ lasers and the developing of computer simulation of MPE process in a strong IR laser field. An adequate description of MPE process needs a detailed information about vibrational-rotational spectra of excited vibrational states of polyatomic molecules. The additional information which is necessary to distinguish between the contributions of different multiphoton transitions into MPE and MPD spectra can be obtained with the use of nonlinear spectroscopy techniques. That is why the results presented in this paper were obtained by four experimental techniques: (1) spectral measurements of absorbed energy; (2) spectral measurements of dissociation yield; (3) four wave mixing spectroscopy; (4) third harmonic generation spectroscopy.

The second direction deveioped in this work is the computer simulation of MPD and MPE spectra. The advantage of computer simulation is concerned with the ability to calculate with the help of computer the MPD and MPE spectra in details without conducting routine and complicated experiments. Such calculations need a comprehensive set of molecular spectroscopic constants which can be obtained from the available linear spectra of one photon transitions between excited states and overtone spectra of a mode under investigation. The comparison between the calculated and observed IR multiphoton spectra of polyatomic molecules presented in this work justify that digital experiment is a reliable way to obtain information about MPE spectra of different molecules.

\section{ABSORPTION SPECTROSCOPY}

Energy absorbed in the gas was measured opto-acoustically. The gas cell was equipped with opto-acoustic detector. To eliminate the effect of hot bands the gas mixtures were cooled down to $140 \mathrm{~K}$ in a gas cell when investigating DL excitation spectra. The DL excitation spectra were studied by high-pressure continuously tuned $\mathrm{CO}_{2}$ laser with e-beam sustained discharge. This laser was described in Reference 5 . The laser pulse duration was $40 \mathrm{~ns}$, its energy reached $1 \mathrm{~J} \mathrm{~cm}^{2}$. The spectral width of emission was less than $0.03 \mathrm{~cm}^{-1}$, the total range of continuous tuning was $86 \mathrm{~cm}^{-1}$, the increment of tuning was $0.03 \mathrm{~cm}^{-1}$ in contrast with the line tuned TEA $\mathrm{CO}_{2}$ laser increment $1.7 \mathrm{~cm}^{-1}$. The frequency of laser pulse was controlled with Fabri-Perot interferometer and opto-acoustic detector with reference gases $\mathrm{N}_{2} \mathrm{O}$ and $\mathrm{CO}_{2}$.

Figure 1 displays the absorption spectra of $\mathrm{SF}_{6}$ molecules at resonant excitation of triply degenerated $v_{3}$ mode that were published in Reference 6 . The energy fluence of $\mathrm{CO}_{2}$ laser pulse was $0.09 \mathrm{~J} \mathrm{~cm}^{2}$ and $0.27 \mathrm{~J} \mathrm{~cm}^{2}$. The total pressure of gas mixture 


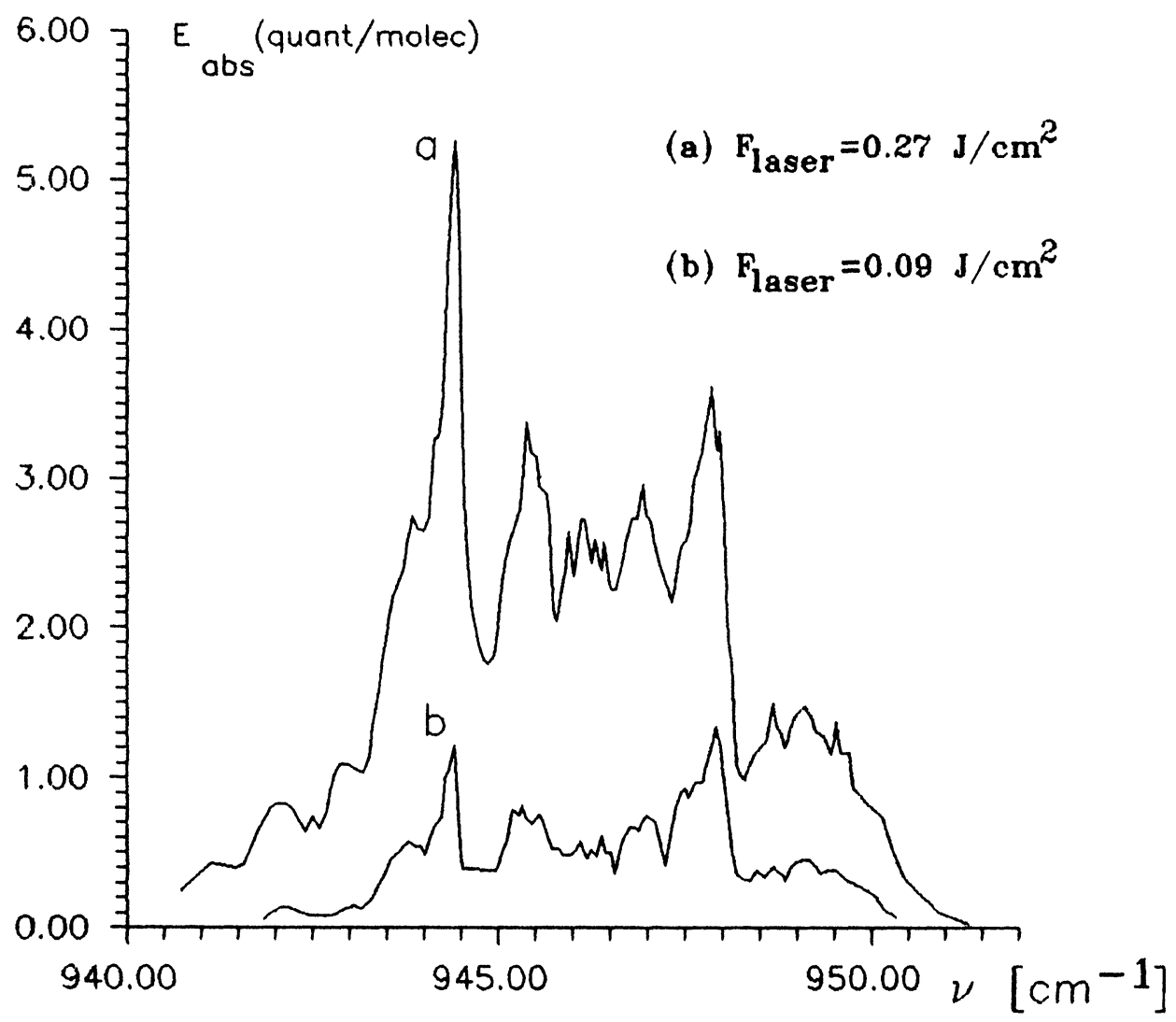

Figure 1 Spectral dependencies of absorbed energy at one frequency excitation of $\mathrm{SF}_{6}$.

$\mathrm{SF}_{6}: \mathrm{Xe}=1: 10$ was 0.3 Torr. The absorbed energy was measured opto-acoustically and calibrated with the help of transmittance measurements.

The spectra reveal a peak of $947.9 \mathrm{~cm}^{-1}$ which is easy to assign as Q-branch of fundamental transition $\left(0 \mathrm{~A}_{1 \mathrm{~g}}-1 \mathrm{~F}_{1 \mathrm{u}}\right)$. The spectra display also the prominent absorption peak at $944.46 \mathrm{~cm}^{-1}$ which is due to two photon transition $0 \mathrm{~A}_{1 \mathrm{~g}}-2 \mathrm{~A}_{1 \mathrm{~g}}$. Indeed, the excited vibrational states of $v_{3}$ mode are split into sublevels due to intramode anharmonic coupling. The $\mathrm{v}=2$ state is split into three sublevels. The anharmonicity constants of $v_{3}$ mode of $\mathrm{SF}_{6}$ are well known from literature. ${ }^{7-10}$ The calculated position of the peak of two photon transition from ground $\mathrm{v}=0 \mathrm{~A}_{1 \mathrm{~g}}$ symmetry vibrational state to $\mathrm{v}=2 \mathrm{~A}_{1 \mathrm{~g}}$ symmetry sublevel is in a good agreement with observed one. Two other sublevels of $E_{g}$ and $F_{2 g}$ symmetry of $v=2$ vibrational state are perturbed due to the vibration-rotation coupling. The peaks in the absorption spectra concerned with $Q$-branches of transitions $0 \mathrm{~A}_{1 \mathrm{~g}}-2 \mathrm{E}_{\mathrm{g}}$ and $0 \mathrm{~A}_{1 \mathrm{~g}}-2 \mathrm{~F}_{2 \mathrm{~g}}$ are braodened due to this coupling. Their positions would be $945.8 \mathrm{~cm}^{-1}\left(\mathrm{E}_{\mathrm{g}}\right.$ sublevel) and $948.4 \mathrm{~cm}^{-1}\left(\mathrm{~F}_{2 \mathrm{~g}}\right.$ sublevel $)$ if there were not vibration-rotation coupling. So the broad peaks near $945.41 \mathrm{~cm}^{-1}$ and $948.7 \mathrm{~cm}^{-1}$ may be assigned as two photon $0 \mathrm{~A}_{1 \mathrm{~g}}-2 \mathrm{E}_{\mathrm{g}}$ and $0 \mathrm{~A}_{1 \mathrm{~g}}-2 \mathrm{~F}_{2 \mathrm{~g}}$ transitions. 
The two frequency technique was used to investigate spectra of transitions between excited states of $v_{3}$ mode of $\mathrm{SF}_{6}$. First laser pulse of 4 atm pressure TEA $\mathrm{CO}_{2}$ laser excited $\mathrm{SF}_{6}$ molecules. Second laser pulse of continuously tuned high pressure $\mathrm{CO}_{2}$ laser was used to probe absorption concerned with the excited molecules. The gas mixture $\mathrm{CO}_{2}: \mathrm{N}_{2}: \mathrm{He}=1: 1: 6$ pressure $4 \mathrm{~atm}$ in the first laser provided the range of continuous tuning $\pm 0.25 \mathrm{~cm}^{-1}$ near $10 \mathrm{P} 16$ line. The frequency of first pulse was chosen to be $947.9 \mathrm{~cm}^{-1}$ in resonance with Q-branch of fundamental transition. The frequency of second pulse was varied in the range $940-944 \mathrm{~cm}^{-1}$. Both pulses were synchronized with zero time delay. The fluctuations of delay time were $\pm 20 \mathrm{~ns}$. The energy fluence of each pulse was $0.1 \mathrm{~J} \mathrm{~cm}^{2}$. The spectrum is shown in Figure 2. Two peaks in the spectrum were assigned as Q-branches of vibrational transitions $1 F_{1 \mathrm{u}}-2 \mathrm{~A}_{1 \mathrm{~g}}$ and $1 \mathrm{~F}_{1 \mathrm{u}}-2 \mathrm{E}_{\mathrm{g}}$ with unresolved rotational structure. The position of $1 \mathrm{~F}_{1 \mathrm{u}}-2 \mathrm{~A}_{1 \mathrm{~g}}$ peak in the spectrum is $940.5 \mathrm{~cm}^{-1}$ and $1 \mathrm{~F}_{1 \mathrm{u}}-2 \mathrm{E}_{\mathrm{g}}$ peak is $943.2 \mathrm{~cm}^{-1}$. The shifts of both peaks from fundamental frequency are twice by comparison with the shifts of two photon peaks $0 \mathrm{~A}_{1 \mathrm{~g}}-2 \mathrm{~A}_{1 \mathrm{~g}}$ and $0 \mathrm{~A}_{1 \mathrm{~g}}-2 \mathrm{E}_{\mathrm{g}}$ in Figure 1. The peak of $1 \mathrm{~F}_{1 \mathrm{u}}-2 \mathrm{E}_{\mathrm{g}}$ transition is broad due to rotation-vibration coupling between $2 \mathrm{E}_{\mathrm{g}}$ and $2 \mathrm{~F}_{2 \mathrm{~g}}$ sublevels.

Figure 3 shows the IR multiphoton absorption spectra of $\mathrm{CF}_{3} \mathrm{Br}$ investigated at gas pressure 0.3 Torr. The spectrum shown in Figure 3a was investigated at one frequency excitation and published in Reference 11. It proved possible to assign main

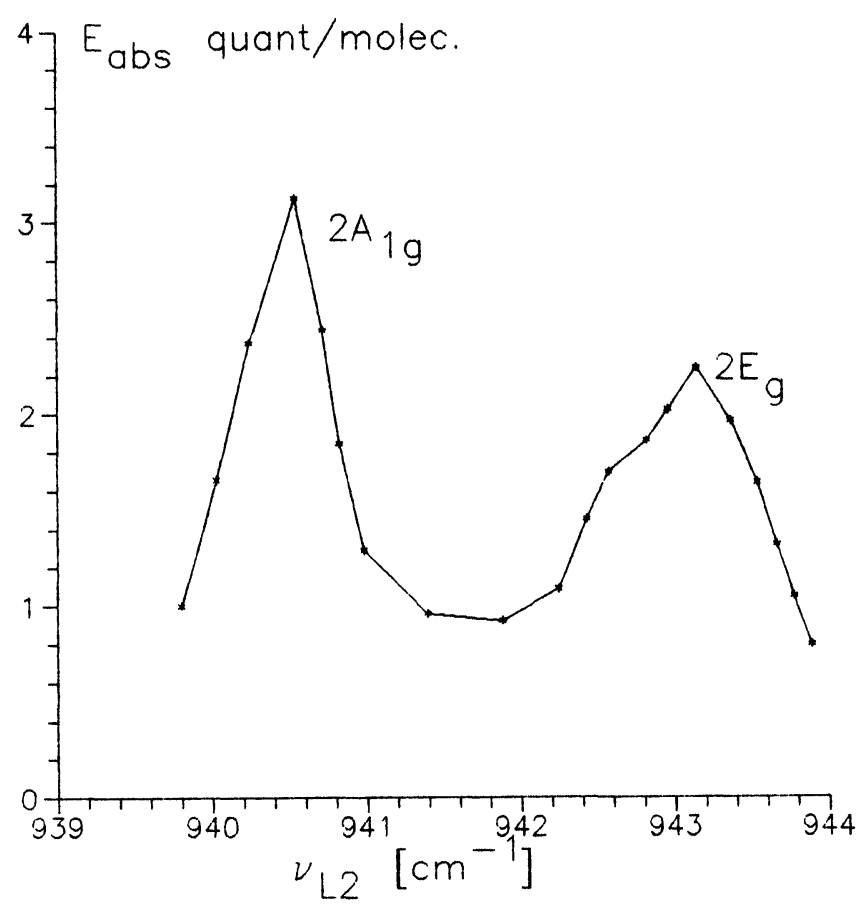

Figure 2 Absorbed energy spectra at two frequency excitation of $\mathrm{SF}_{6}$. 

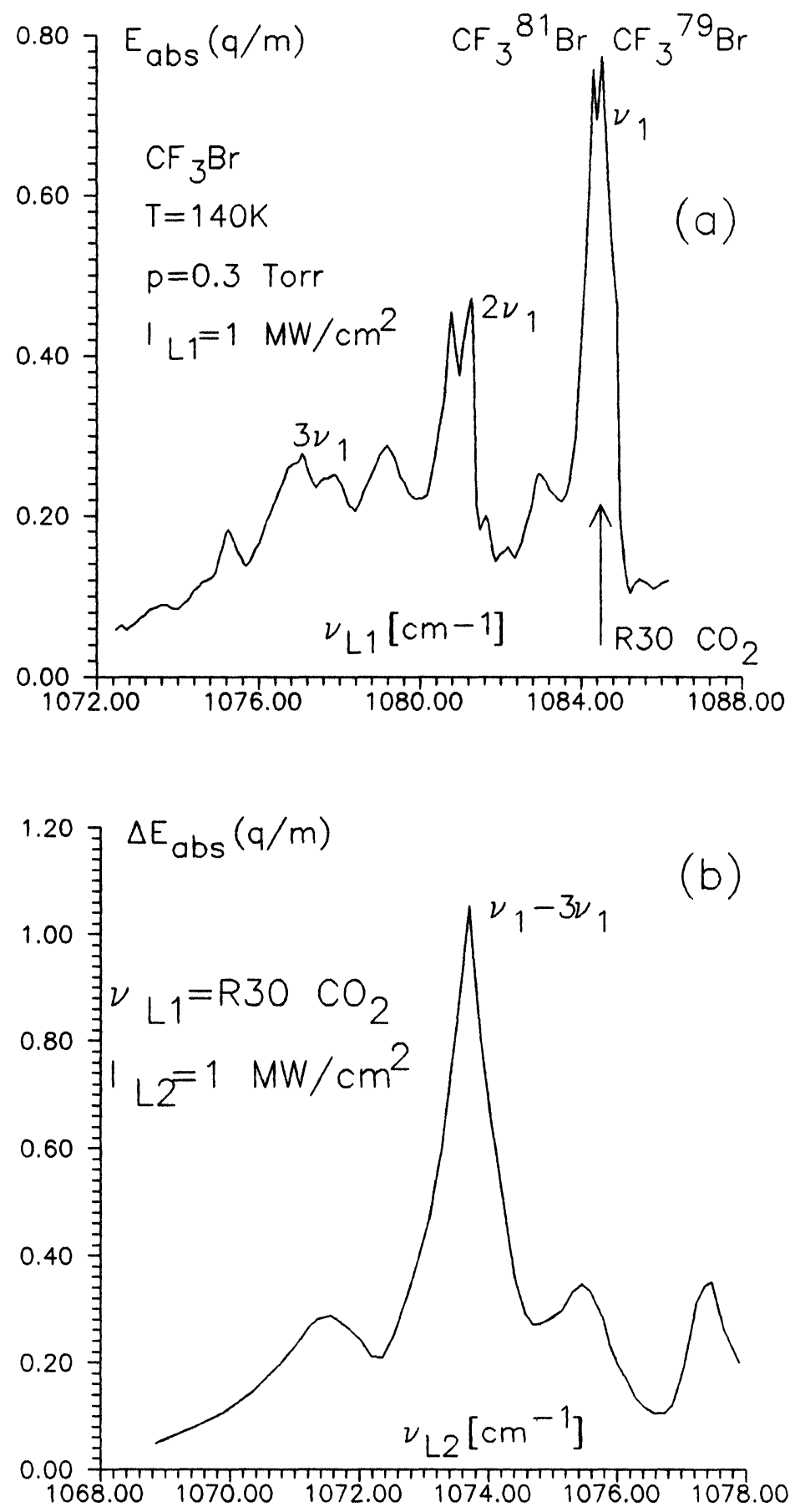

Figure 3 Absorption spectra of $\mathrm{CF}_{3} \mathrm{Br}$. One (a) and two (b) frequency excitation. 
peaks in this spectrum. The doublets with lines separated by $\Delta=0.26 \mathrm{~cm}^{-1}$ are owing to isotopic species $\mathrm{CF}_{3}{ }^{79} \mathrm{Br}$ and $\mathrm{CF}_{3}{ }^{81} \mathrm{Br}$. The successive doublets separated by $3.34 \mathrm{~cm}^{-1}$ are the peaks of one, two and three photon absorption lines the three photon peak being a weak. Both values are in a good agreement with the isotopic shift of $v_{1}$ mode of $\mathrm{CF}_{3} \mathrm{Br} \Delta=0.242 \mathrm{~cm}^{-1}$ and the anharmonicity constant $\mathrm{X}_{11}=3.37 \mathrm{~cm}^{-1} \cdot{ }^{12-14}$

Q-branch of fundamental transition of $v_{1}$ mode of $\mathrm{CF}_{3} \mathrm{Br}$ is in a resonance with 9R30 line of $\mathrm{CO}_{2}$ laser. Spectrum of Figure 3b shows the result of two frequency experiment when $\mathrm{CF}_{3} \mathrm{Br}$ gas was excited by the pulse of atmospheric TEA $\mathrm{CO}_{2}$ laser operating at 9R30 line and then the absorption spectrum of excited molecules was investigated with continuously tuned $\mathrm{CO}_{2}$ laser. The two pulses were synchronized with zero delay time. Both TEA $\mathrm{CO}_{2}$ laser pulse and probing pulse intensities were $1 \mathrm{MW} \mathrm{cm}{ }^{2}$. The spectrum shows the peak assigned as two photon transition $1 v_{1}-3 v_{1}$. The peak has no noticeable isotopic structure due to selective excitation of one isotopic species by the first laser pulse.

The peaks observed in the IR multiphoton absorption spectra of $\mathrm{SF}_{6}$ and $\mathrm{CF}_{3} \mathrm{Br}$ have a width about $0.3 \mathrm{~cm}^{-1}$. The width of peaks did not depend on laser pulse

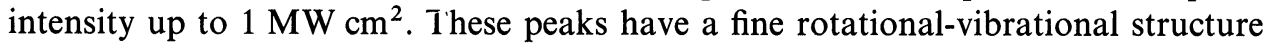
which can be observed with resolution of $1 \mathrm{MHz} .^{7,8}$ The observed in our experiments width $0.3 \mathrm{~cm}^{-1}$ is due to inhomogeneous broadening. It is clear that one can use these peaks in IR multiphoton excitation spectra to achieve a high selectivity of MPE even in the excitation of molecules of heavy isotopes. The deep cooling of a gas leading to reducing of inhomogeneous broadening may be used to increase the selectivity of excitation. The two frequency excitation has obvious advantage of multiplication this selectivity.

The following experiments were done to investigate the absorption spectra in QC region of polyatomic molecules. The results of these experiments were earlier published in Reference 15. The absorption spectra were investigated by the two frequency technique. $\mathrm{SF}_{6}$ gas at room temperature was excited by $10 \mathrm{P} 20$ TEA $\mathrm{CO}_{2}$ laser pulse with varied energy fluence in the range $0.5-2.5 \mathrm{~J} \mathrm{~cm}^{2}$. The linear absorption spectra of molecules excited into quasicontinuum was probed by second laser pulse. To probe linear absorption ${ }^{12} \mathrm{CO}_{2},{ }^{13} \mathrm{CO}_{2}$ and $\mathrm{NH}_{3}$ lasers were used. The spectral region $770-950 \mathrm{~cm}^{-1}$ was covered. The absorption cross section $\sigma$ was measured by transmittance technique. The energy fluence of second pulse was chosen to satisfy the linearity condition and did not exceed $1 \mathrm{~mJ} / \mathrm{cm}^{2}$. The time delay between two pulses was $1 \mu \mathrm{s}$. The spectral dependencies of $\sigma$ versus laser frequency are shown in Figure 4 for three levels of excitation of molecules by first laser pulse: $E_{a b s}=8.4$, 13.4 , and 25 quanta per molecule.

There is only alone structureless peak in the spectra. This peak is shifted due to anharmonicity to the long wave side from the fundamental transition of $v_{3}$ mode. Both shift and width of a peak rise linearly with increasing of absorbed energy. In fact observed peak is due to overlapping of the spectra of successive transitions in QC. The changes in the peak position and width are due to changes in the population distribution in QC. A simple and reasonable model make it possible to estimate the characteristics of individual transition in QC. Let assume that line shape of individual 


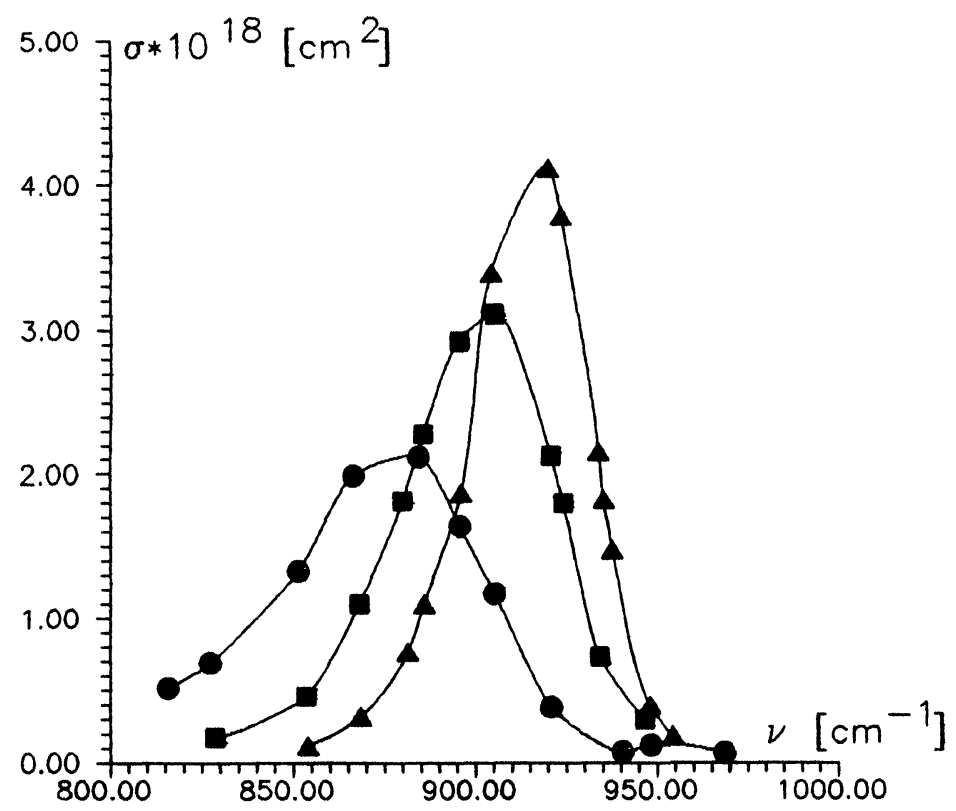

Figure 4 Linear absorption spectra of $\mathrm{SF}_{6}$ in quasicontinuum. $\mathrm{E}_{\mathrm{abs}}=8.4 \mathrm{q} / \mathrm{m}$-triangles; $\mathrm{E}_{\mathrm{abs}}=$ $13.4 \mathrm{q} / \mathrm{m}$-squares; $\mathrm{E}_{\text {abs }}=25 \mathrm{q} / \mathrm{m}$-circles.

transition in QC has Lorentz profile:

$$
\sigma(v, \mathrm{v} \rightarrow \mathrm{v}+1)=\frac{\mathrm{A}_{01} \mathrm{X}_{2} \mathrm{v}}{\pi\left[\left(v-v_{0}-\mathrm{X}_{1} \mathrm{v}\right)^{2}+\left(\mathrm{X}_{2} \mathrm{v}\right)^{2}\right]}
$$

where $\mathrm{A}_{01}$ is integral cross section of vibrational mode; $v_{0}$ is frequency of fundamental transition; shift $X_{1} v$ and half-width $X_{2} v$ increase linearly with increasing of the number $\mathrm{v}$ of the level in the ladder of states populated by laser pulse. Let population distribution over $\mathrm{v}$ is thermal like:

$$
\mathrm{n}(\mathrm{v})=\mathrm{v}^{\mathrm{s}-1} \exp (-\mathrm{sv} / \Theta) /[\Theta(\mathrm{s}-1) !]
$$

where $s$ is the number of vibrational degrees of freedom in $\mathrm{QC} ; \Theta=\mathrm{kT} / \mathrm{hcv}$, T being the effective temperature of the vibrational distribution. The modeling of observed spectra yields $\mathrm{A}_{01} \approx 1.7 \cdot 10^{-16} \mathrm{~cm}, \mathrm{~s} \approx 10, \mathrm{X}_{1} \approx-3.1 \mathrm{~cm}^{-1}, \mathrm{X}_{2} \approx 0.4 \mathrm{~cm}^{-1}$. The first conclusion is that measured integral cross section is equal to that of the fundamental transition. ${ }^{16}$ The number of degrees of freedom $s$ involved in $\mathrm{QC}$ formation is less than the total number of vibrational degrees of freedom in $\mathrm{SF}_{6}$ $\mathrm{s}_{\text {total }}=15$. Apparently this fact can be explained by so called "freezing" of high frequency modes participating in QC formation. So the statistical weight of high frequency modes decreases when $\mathrm{kT}$ is less than hcv. The very interesting result is that broadening $\mathrm{X}_{2} \mathrm{v}$ of transitions in $\mathrm{QC}$ is small by comparison with the shift $\mathrm{X}_{1} \mathrm{v}$. 


\section{DISSOCIATION SPECTROSCOPY}

The spectral structure of discrete level excitation appears in the dissociation spectra. ${ }^{17}$ This fact is of great importance for the selectivity of multiphoton dissociation of polyatomic molecules.

The experiments on dissociation spectroscopy were carried out with gases cooled in supersonic jet. We investigated IR MPD spectra of $\mathrm{SF}_{6}$ and $\mathrm{OsO}_{4}$ molecules. The gasdynamic cooling makes it possible to obtain low rotational temperature and thus reduce the inhomogeneous broadening of multiphoton transitions. The rotational temperature of molecules cooled in supersonic jet was estimated with diode spectroscopy measurements to be $\mathrm{T}_{\text {rot }} \approx 30 \mathrm{~K}$ and vibrational temperature $\mathrm{T}_{\mathrm{vib}} \approx 100 \mathrm{~K}$.

The dissociation yield was measured by the luminescence technique. The products of $\mathrm{SF}_{6} \mathrm{MPD}$ process are $\mathrm{SF}_{5}$ radicals and $\mathrm{F}$ atoms. The chemical reaction of $\mathrm{F}$ atoms with hydrogen molecules was used to obtain HF-luminescence signal proportional to the dissociation yield of $\mathrm{F}$ atoms :

$$
\mathrm{F}+\mathrm{H}_{2} \rightarrow \mathrm{H}+\mathrm{HF}^{*}, \quad \mathrm{HF}^{*} \rightarrow \mathrm{HF}+\mathrm{h} v(3 \mu \mathrm{m}) .
$$

The dissociation of $\mathrm{OsO}_{4}$ molecules is accompanied with visible luminescence. ${ }^{18}$

The dissociation of $\mathrm{SF}_{6}$ molecules was performed with the help of three $\mathrm{CO}_{2}$ laser pulses. Two pulses were used to study the process of DL excitation in the course of MPD. Third pulse was applied to dissociate molecules excited into the quasicontinuum. The frequency of first pulse was close to the fundamental frequency of $v_{3}$ of $\mathrm{SF}_{6}$. The frequency of second pulse was chosen to be in resonance with transitions between excited states of $v_{3}$ mode. The frequency of third pulse was shifted to the longwave side from fundamental $v_{3}$ mode transition to optimize the excitation process in QC region. It was possible to investigate as the spectra of multiphoton transitions from ground vibrational state and the spectra of transitions between excited vibrational states.

Figure 5 shows the dissociation spectra of $\mathrm{SF}_{6}$. The stagnation pressure of $\mathrm{SF}_{6}: \mathrm{H}_{2}=1: 20$ mixture in the nozzle receiver chamber was 250 Torr. The pulsed slit nozzle dimensions were $0.03 \times 3 \mathrm{~cm}$. The nozzle chamber was pumped out to pressure $10^{-6}$ Torr. The $\mathrm{PbS}$ detector was used to measure HF luminescence signal. Two TEA $\mathrm{CO}_{2}$ lasers and one continuously tuned high pressure $\mathrm{CO}_{2}$ laser were utilized in experiments. Three laser pulses were synchronized. Time delay between first and second pulses was chosen to be zero the fluctuations of time delay being in the range $\pm 20 \mathrm{~ns}$. The time delay of third pulse was $500 \mathrm{~ns}$. Third laser operated with ${ }^{13} \mathrm{CO}_{2}$ gas mixture to obtain laser pulse frequency near $900 \mathrm{~cm}^{-1}$ that is optimal in the course of $\mathrm{SF}_{6}$ excitation in $\mathrm{QC}$ region.

Two combinations of laser frequencies were used to investigate DL spectra. First combination with TEA CO $\mathrm{CO}_{2}$ lasers operating at $10 \mathrm{P} 34$ line of ${ }^{12} \mathrm{CO}_{2}\left(v_{\mathrm{L} 2}=931 \mathrm{~cm}^{-1}\right)$ and $10 \mathrm{P} 30$ of ${ }^{13} \mathrm{CO}_{2}\left(\mathrm{v}_{\mathrm{L} 3}=888 \mathrm{~cm}^{-1}\right)$ was chosen to investigate with continuously tuned laser pulse the spectra of multiphoton transitions of $\mathrm{SF}_{6}$ from the ground vibrational state to the excited states. Second combination with two TEA $\mathrm{CO}_{2}$ lasers operating at $947.9 \mathrm{~cm}^{-1}$ near $10 \mathrm{P} 16$ of ${ }^{12} \mathrm{CO}_{2}$ and $10 \mathrm{P} 12$ line of ${ }^{13} \mathrm{CO}_{2}$ 

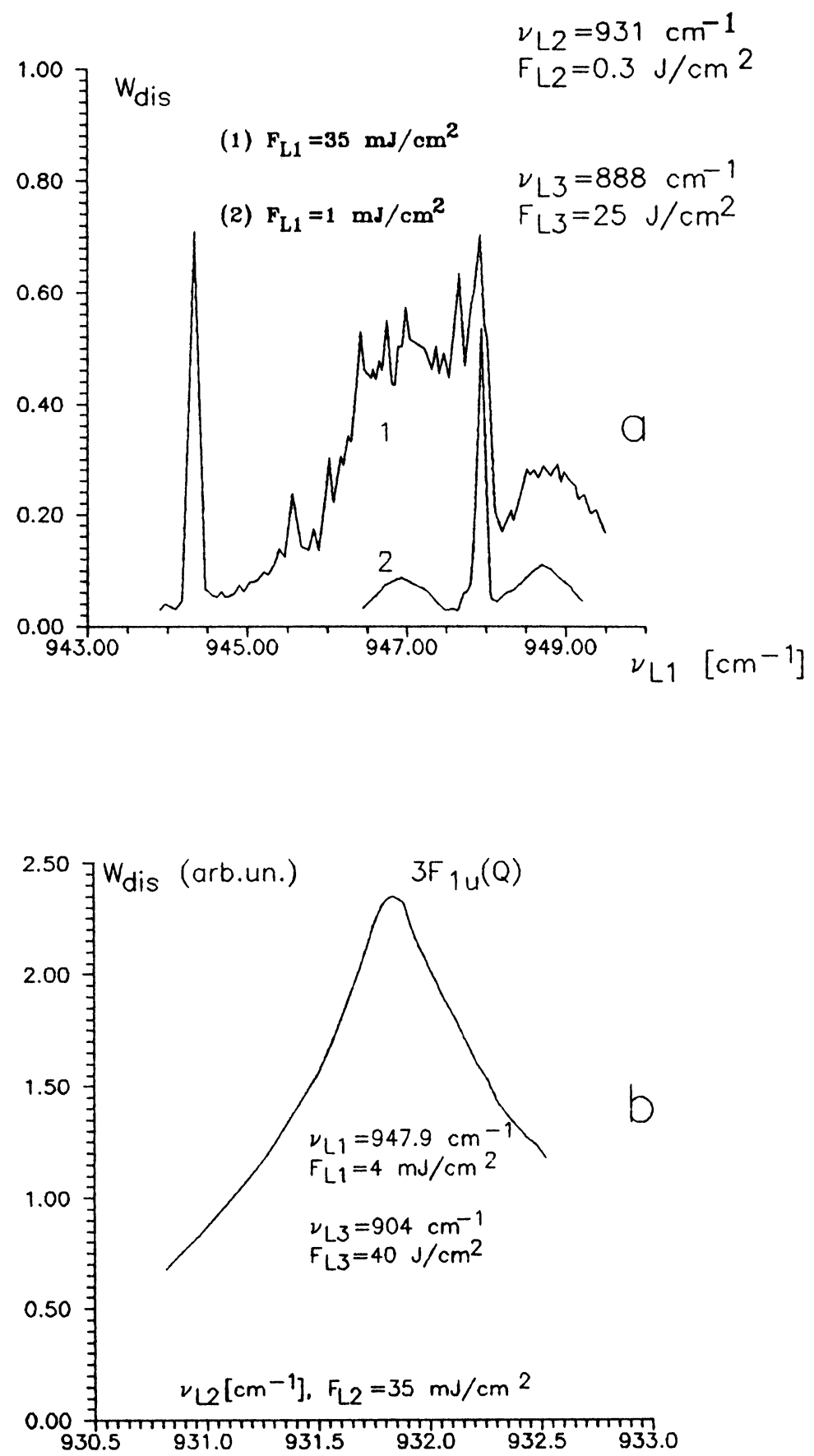

Figure 5 Dissociation spectra of $\mathrm{SF}_{6}$ with first laser pulse tuned (a) and second laser pulse tuned (b). 
$\left(\mathrm{v}_{\mathrm{L} 3}=904 \mathrm{~cm}^{-1}\right)$ were chosen to investigate with continuously tuned high pressure laser pulse the spectra of transitions between excited states of $\mathrm{SF}_{6}$. Increasing of the energy fluences of three pulses resulted in a saturation of HF-luminescence signal. The saturation limit of HF-signal was assumed to correspond to $100 \%$ dissociation yield.

Figure 5a displays results obtained with first combination of laser pulse frequencies. The most striking result is that the dissociation spectrum reveals the structure of the fundamental vibrational transition of $v_{3}$ mode of $\mathrm{SF}_{6}$ at energy fluence of continuously tuned laser pulse $1 \mathrm{~mJ} / \mathrm{cm}^{2}$. It became possible to observe this spectrum due to optimal choose of frequencies of second and third laser pulses. Increasing of first laser pulse energy fluence leads to the appearance of two photon peaks in the dissociation spectrum. These peaks are seen in the spectrum obtained at first laser pulse energy fluence $35 \mathrm{~mJ} / \mathrm{cm}^{2}$. The most prominent peak is those concerned with two photon transition $0 \mathrm{~A}_{1 \mathrm{~g}}-2 \mathrm{~A}_{1 \mathrm{~g}}$ at frequency $944.46 \mathrm{~cm}^{-1}$. It is very important from isotope separation point of view that the dissociation yield at maximum is close to $100 \%$ for both spectra.

The cooling of $\mathrm{SF}_{6}$ in supersonic jet resulted in the narrowing of two photon peaks. Indeed, the width of $0 \mathrm{~A}_{1 \mathrm{~g}}-2 \mathrm{~A}_{1 \mathrm{~g}}$ peak that was measured with resolution $\pm 0.03 \mathrm{~cm}^{-1}$ is not more than $0.1 \mathrm{~cm}^{-1}$ at cooling in supersonic jet. The width of the same peak measured in the absorption spectra obtained at $\mathrm{T}=140 \mathrm{~K}$ is approximately $0.3 \mathrm{~cm}^{-1}$. Low rotational temperature results in the reducing of a width of other Q-branches of two photon transitions as well. The main reason is concerned with the depopulation of rotational states with high values of rotational quantum number $\mathbf{J}$ at low rotational temperature. Thus the inhomogeneous broadening due to rotation-vibration coupling in the $\mathrm{v}=2$ vibrational state is reduced. Q-branch of two photon transition $0 \mathrm{~A}_{1 \mathrm{~g}}-2 \mathrm{E}_{\mathrm{g}}$ is seen in the dissociation spectra as a contrast and narrow peak with frequency position $945.6 \mathrm{~cm}^{-1}$. Q-branch of two photon transition $0 \mathrm{~A}_{1 \mathrm{~g}}-2 \mathrm{~F}_{2 \mathrm{~g}}$ is not seen in the spectrum as narrow peak. Two reasons can be assumed to explain this fact. First is that it needs cooling to the temperatures less than $30 \mathrm{~K}$ to reduce the inhomogeneous width of this Q-branch. Second is that Q-branch of three photon transition $0 \mathrm{~A}_{1 \mathrm{~g}}-3 \mathrm{~A}_{2 \mathrm{u}}$ which can be observed at $948.6 \mathrm{~cm}^{-1}$ overlaps this two photon Q-branch that results in the broad structureless peak near $949 \mathrm{~cm}^{-1}$ which is seen in the dissociation spectra. The appearance of some peaks in the region $946-947 \mathrm{~cm}^{-1}$ which could not be assigned as two photon is the evidence that there are multiphoton transitions (more than two photon) with small detuning off fundamental $v_{3}$ frequency in the dissociation spectrum.

Figure $5 \mathrm{~b}$ displays results with second combination of laser frequencies. First $4 \mathrm{~atm}$ TEA $\mathrm{CO}_{2}$ laser pulse was in the resonance with Q-branch of fundamental transition. Its frequency was tuned to $947.9 \mathrm{~cm}^{-1}$. The pulse of continuously tunable high pressure $\mathrm{CO}_{2}$ laser was used as second pulse. Its frequency was varied in the region 931-933 $\mathrm{cm}^{-1}$. Shown in Figure 6 is a contrast peak observed near the frequency $931.8 \mathrm{~cm}^{-1}$ in the dissociation spectrum. This peak is obviously concerned with transitions between excited states of $v_{3}$ mode of $\mathrm{SF}_{6}$. Indeed, the combination of the two frequencies $v_{\mathrm{L} 1}=947.9 \mathrm{~cm}^{-1}$ and $v_{\mathrm{L} 2}=931.8 \mathrm{~cm}^{-1}$ is in resonance with Q-branch of two photon transition $1 \mathrm{~F}_{1 \mathrm{u}}-3 \mathrm{~F}_{1 \mathrm{u}}$ as it follows from data on $3 v_{3}$ overtone 

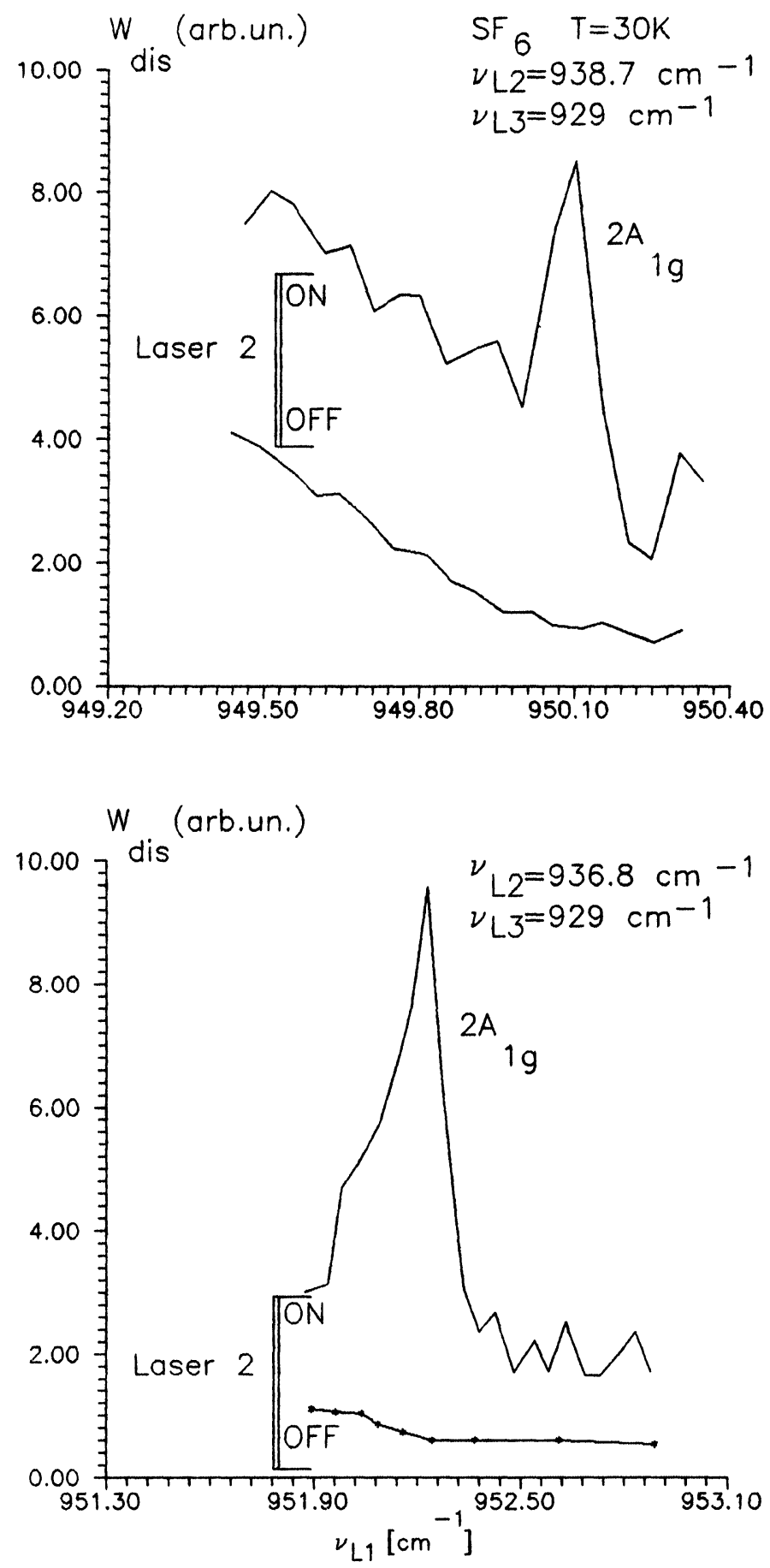

Figure 6 Bichromatic excitation of $\mathrm{SF}_{6}$. 
spectra. ${ }^{9}$ Calculations of $v_{3}$ mode ladder show (see below) that at the same time $v_{\mathrm{L} 2}$ is in resonance with Q-branch of one photon transitions $3 \mathrm{~F}_{1 \mathrm{u}}-4 \mathrm{~A}_{1 \mathrm{~g}}$ and $3 \mathrm{~F}_{1 \mathrm{u}}-4 \mathrm{E}_{\mathrm{g}}$ (energy gap between vibrational sublevels $A_{1 g}$ and $E_{g}$ of $v=4$ is very small). So this combination is an optimal one for excitation from $\mathrm{v}=1$ state of $v_{3}$ up to $\mathrm{v}=4$ state.

Thus, experiments on three frequency multiphoton dissociation of $\mathrm{SF}_{6}$ cooled in supersonic jet showed that there are narrow resonances in the IR multiphoton spectra concerned with multiphoton transitions in DL ladder. It is possible to take advantage of these resonances to investigate the structure of vibrational-rotational spectra of excited vibrational states of polyatomic molecules. These resonances demonstrate a way to reach high selectivity and high efficiency of isotope separation by means of MPD via multifrequency laser excitation.

\section{BICHROMATIC EXCITATION}

Employing of two frequencies in the course of DL excitation make it possible to perform a bichromatic (two color) excitation of two photon transitions. Bichromatic excitation of two photon transitions with the help of two simultaneously applied laser pulses may be useful from the IR multiphoton spectroscopy point of view. Indeed, if a line tuned lasers are used for DL excitation, one deals with limited number $\mathrm{N}$ of these lines. Employing of bichromatic excitation make it possible to chose some of available $\mathrm{N} \cdot(\mathrm{N}+1) / 2$ combinations which are in resonance with two photon transitions of interest. Another advantage is concerned with the possibility to investigate IR multiphoton spectra anaccessible with one frequency DL excitation.

Figure 6 shows dissociation spectra of $\mathrm{SF}_{6}$ at bichromatic excitation of discrete levels. First and second laser pulses were synchronized with zero time delay. Those frequencies $v_{\mathrm{L} 1}$ and $v_{\mathrm{L} 2}$ were chosen to satisfy two photon resonance condition:

$$
v_{\mathrm{L} 1}+v_{\mathrm{L} 2}=\left(\mathrm{E}\left(\mathrm{v}=2, \mathrm{~A}_{1 \mathrm{~g}}\right)-\mathrm{E}\left(\mathrm{v}=0, \mathbf{A}_{1 \mathrm{~g}}\right)\right) / \mathrm{hc}
$$

Two photon peak in the dissociation spectra obtained by varying the frequency of first pulse throughout appropriate region was observed for two frequencies of second pulse. Two frequencies of second pulse were $938.7 \mathrm{~cm}^{-1}(10 \mathrm{P} 26)$ and $936.8 \mathrm{~cm}^{-1}$ (10P28). Frequency of third pulse was $929 \mathrm{~cm}^{-1}$ (10P36). Intensities of three pulses were $\sim 2, \sim 0.2$ and $\sim 70 \mathrm{MW} / \mathrm{cm}^{2}$ respectively. The difference between the spectra with second pulse on/off demonstrates that observed peak is due to bichromatic excitation. The altering of second pulse frequency results in changing of peak position in accordance with relation (4) that Figure $6 \mathrm{a}$ and Figure $6 \mathrm{~b}$ demonstrate. The observed shift of the peak satisfies (4) relation. This is a convincing evidence that this peak is due to two photon transition.

Figure 7 shows the spectrum of bichromatic excitation of $\mathrm{OSO}_{4}$ cooled in a supersonic jet. The mixture of $\mathrm{OsO}_{4}: \mathrm{He}=1: 250$ was cooled down to rotational temperature $T_{\text {rot }}=30 \mathrm{~K}$. The frequency of first pulse $v_{\mathrm{L} 1}$ was varied throughout the region $940 \mathrm{~cm}^{-1}-955 \mathrm{~cm}^{-1}$. The frequency of second pulse was $v_{\mathrm{L} 2}=969.1 \mathrm{~cm}^{-1}$ (10R10). The spectrum is represented as the dependence of the ratio of luminescence signal with the second pulse on to the signal with second pulse off versus reduced 


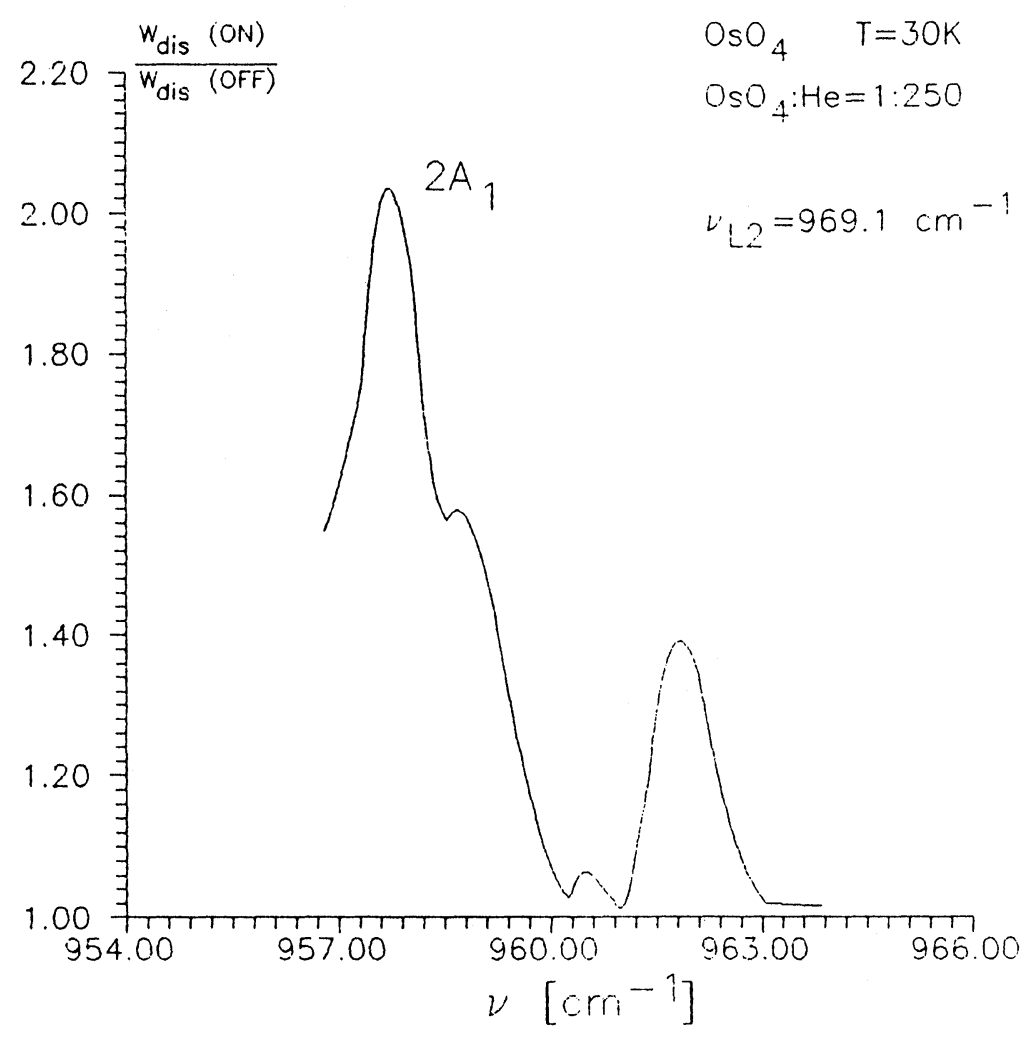

Figure 7 Bichromatic excitation of $\mathrm{OsO}_{4}$.

frequency $v=\left(v_{\mathrm{L} 1}+v_{\mathrm{L} 2}\right) / 2$. The reduced frequency was tuned from $954.6 \mathrm{~cm}^{-1}$ to $962.1 \mathrm{~cm}^{-1}$. This frequency region lies just between $\mathrm{P}$ and $\mathrm{R}$ generation branches of common ${ }^{12} \mathrm{CO}_{2}$ laser and is anaccessible for one frequency study of multiphoton transition in $v_{3}$ mode of $\mathrm{OsO}_{4}$. The position of two photon peak in the observed spectrum is in a good agreement with the frequency of first overtone of $v_{3}$ of $\mathrm{OsO}_{4}$, measured in Reference 19. It is important from the isotope selective dissociation point of view that $\mathrm{OSO}_{4}$ is an example of a molecule with heavy atom. The observed spectrum demonstrates therefore the availability of selective dissociation of molecules with heavy atom or with small isotopic shift of vibrational frequency.

\section{NONLINEAR SPECTROSCOPY TECHNIQUES}

Two nonlinear techniques as degenerate four wave mixing and third harmonic generation were utilized to investigate the excited vibrational state spectra of polyatomic molecules. These techniques have an advantage in the coarse of investigation of second or third vibrational level. As absorption and dissociation 
spectroscopy yields the spectra with overlapping peaks of one, two and more photon transitions. Nonlinear spectroscopy makes it possible to observe only spectra concerned with two or three photon transitions. So it has an advantage in the investigation of spectra of certain vibrational level.

Degenerate four wave mixing is a process of coherent generation of light wave when pumping of a media by three coherent light waves with all waves being of the same frequency. To satisfy coherence condition the wave vectors of four waves must obey the relation

$$
\overrightarrow{\mathrm{k}}_{1}+\overrightarrow{\mathrm{k}}_{2}=\overrightarrow{\mathrm{k}}_{3}+\overrightarrow{\mathrm{k}}_{4} \text {. }
$$

This relation means that irradiating of a media with three waves in such a manner that two of them (pump waves) are propagating in opposite directions $\left(\overrightarrow{\mathrm{k}}_{1}=\overrightarrow{\mathrm{k}}_{2}\right)$ results in a generation of fourth wave (signal wave) propagating in opposite direction to third (probe) wave: $\overrightarrow{\mathrm{k}}_{3}=-\overrightarrow{\mathrm{k}}_{4}$. So it is very easy to discriminate the signal wave in experiment.

Resonant interaction of laser fields with molecules makes it possible to perform degenerate four wave mixing spectroscopy (DFWMS) of first and second excited vibrational states of IR active modes. In the case of resonant interaction of laser fields with IR active mode two ways of DFWM are possible. ${ }^{20,21}$ These ways are shown in the energy diagrams of Figure 8.

In a first case shown in Figure 8 the polarization is defined by the relation

$$
\overrightarrow{\mathrm{P}}_{4}=\chi_{1} \cdot\left(\overrightarrow{\mathrm{E}}_{1} \cdot \overrightarrow{\mathrm{E}}_{3}^{*}\right) \cdot \overrightarrow{\mathrm{E}}_{2}
$$

where $\overrightarrow{\mathrm{E}}_{1}$ are amplitudes of pump and probe fields, $\overrightarrow{\mathrm{P}}_{4}$ is polarization of a molecule which results in generation of signal wave, $\chi_{1}$ is nonlinear susceptibility of a molecule which depends on the laser frequency in a resonant manner and is proportional to

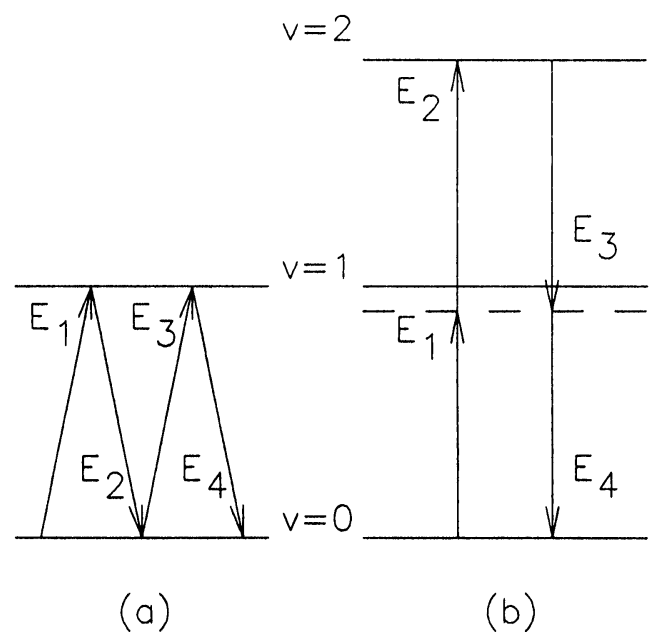

Figure 8 Diagram of level pumping in the process of DFWM. 
$\left[\left(v-v_{01}+\mathrm{i} \cdot \gamma_{01}\right)\right]^{-1}, v_{01}$ is frequency of one photon transition, $\gamma_{01}$ is line width of the transition.

In a second case shown in Figure 8 the polarization is defined by the relation

$$
\overrightarrow{\mathrm{P}}_{4}=\chi_{2} \cdot\left(\overrightarrow{\mathrm{E}}_{1} \cdot \overrightarrow{\mathrm{E}}_{2}\right) \cdot \overrightarrow{\mathrm{E}}_{3}^{*}
$$

where nonlinear susceptibility $\chi_{2}$ resonantly depends on the laser frequency and is proportional to $\left[\left(2 \cdot v-v_{02}+\mathrm{i} \cdot \gamma_{02}\right)\right]^{-1}$ near the frequency of two photon transition $v_{02}, \gamma_{02}$ is line width of this transition.

Employing of linearly polarized laser fields with polarization of probe wave perpendicular to polarization of pump waves make it possible to realize the generation of signal wave by second way. As result it is possible to observe the spectra of two photon transition by means of DFWMS.

Figure 9 shows the DFWM spectrum of $\mathrm{SF}_{6}$ cooled in a cell at temperature $\mathrm{T}=140 \mathrm{~K}$. Three waves were produced from one laser pulse by splitting of laser beam with germanium plate (one transmitted and two reflected). The angle between pump and probe waves was $3^{\circ}$. The pump wave intensition were equal. Intensity of probe wave was $I_{\text {probe }}=0.1 \cdot I_{\text {pump }}$. Reflection index of weak probe laser pulse was measured. The spectrum reveals peaks of two photon transitions $0 \mathrm{~A}_{1 \mathrm{~g}}-2 \mathrm{~A}_{1 \mathrm{~g}}$ $\left(944.46 \mathrm{~cm}^{-1}\right), 0 \mathrm{~A}_{1 \mathrm{~g}}-2 \mathrm{E}_{\mathrm{g}}\left(945.6 \mathrm{~cm}^{-1}\right), 0 \mathrm{~A}_{1 \mathrm{~g}}-2 \mathrm{~F}_{2 \mathrm{~g}}$ (peaks near $948 \mathrm{~cm}^{-1}$ ).

The DFWM spectrum has more simple structure than the absorption spectrum

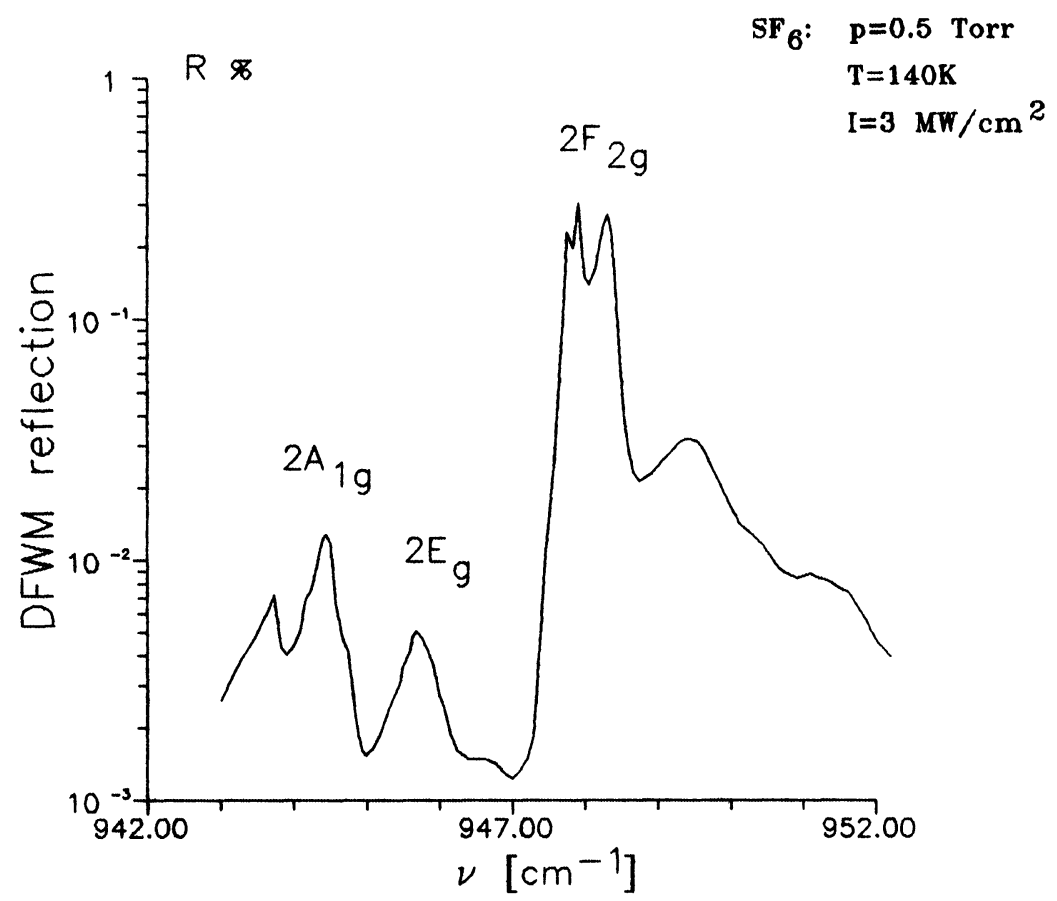

Figure 9 DFWM spectrum of $\mathrm{SF}_{6}$. 
obtained at similar conditions (see Figure 1). There are not peaks due to multiphoton transitions with order of transition more than two in DFWM spectra in contrast to absorption spectra at the same temperature $T=140 \mathrm{~K}$. So it follows that complex structure in the absorption spectra which was observed in the spectral range 946-947 $\mathrm{cm}^{-1}$ is due to three photon transitions and maybe to four photon one.

Another nonlinear IR multiphoton spectroscopy technique utilized to investigate multiphoton spectra is third harmonic generation spectroscopy (THGS). The polarization of molecules resonantly pumped by laser field has a third harmonic contribution which is sensitive to the resonances of laser field with the multiphoton transitions in pumped vibrational mode:

$$
\overrightarrow{\mathrm{P}}_{3 v}=\chi_{3 v} \cdot(\overrightarrow{\mathrm{E}} \cdot \overrightarrow{\mathrm{E}}) \cdot \overrightarrow{\mathrm{E}}
$$

with $\chi_{3 v} \sim\left[\left(v-v_{01}\right) \cdot\left(2 \cdot v-v_{02}\right) \cdot\left(3 \cdot v-v_{03}\right)\right]^{-1} ; v_{01}, v_{02}$ and $v_{03}$ are frequencies of one, two and three photon transition. Resonant enhancement of nonlinear susceptibility makes it possible to conduct the investigation of the spectra of excited vibrational states by means of THGS.

The THG spectrum of $\mathrm{SF}_{6}$ cooled in supersonic jet was obtained with the help of continuously tuned $\mathrm{CO}_{2}$ laser. The $10 \mu \mathrm{m}$ laser radiation transmitted through the jet was filtered out and $3 \mu \mathrm{m}$ third harmonic signal was measured by InSb detector. Figure 10 shows THG spectrum of $\mathrm{SF}_{6}$ at $\mathrm{T}=30 \mathrm{~K}$ obtained at laser intensity

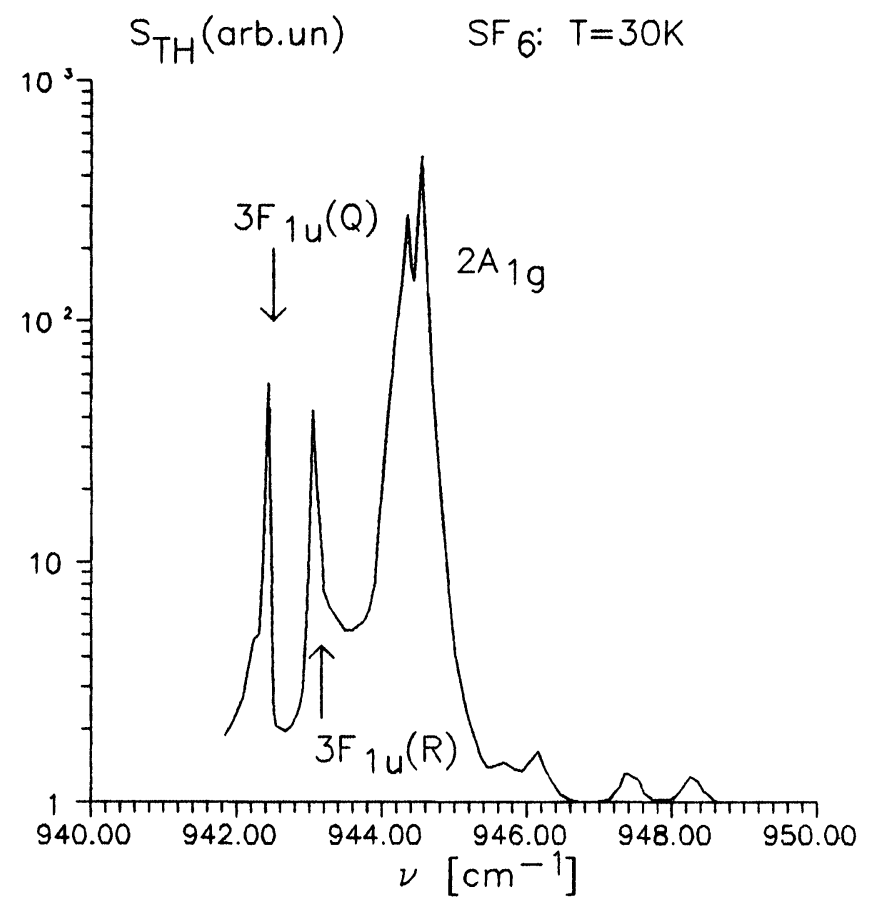

Figure 10 THG spectra of $\mathrm{SF}_{6}$. 
$1 \mathrm{MW} / \mathrm{cm}^{2}$. There are two new peaks in the spectrum by comparison with the dissociation spectrum obtained at similar conditions (see Figure 5). These peaks have frequencies $942.2 \mathrm{~cm}^{-1}$ and $943.03 \mathrm{~cm}^{-1}$. They are shifted to the long wave side from the two photon peak at $944.46 \mathrm{~cm}^{-1}$. The latter is split due to saturation of two photon transition. The dip was observed in the two photon peak when laser frequency was exactly in resonance with this transition. These new peaks can be assigned as $Q$ and $R$ rotational branches of three photon transition $0 \mathrm{~A}_{1 \mathrm{~g}}-3 \mathrm{~F}_{1 \mathrm{u}}$ as it follows from the measurements of second overtone spectra of $\mathrm{SF}_{6} \cdot{ }^{9}$

The nonlinear spectroscopy techniques are the complimentary to the absorption and dissociation spectroscopy of excited vibrational states of polyatomic molecules. These techniques give a reliable assignment of observed peaks as two or three photon transition. The THG spectroscopy is more straightforward in the observation of spectra of third vibrational state of a vibrational mode by comparison with the absorption or dissociation spectroscopy which needs to use two frequency technique to do it.

\section{COMPUTER SIMULATION OF MOLECULAR SPECTRA}

Computer calculations of the excited vibrational state spectra are of great interest for the investigation of excited states of molecules. The calculations make it possible to extract a spectroscopic parameters of a molecule from the spectra observed in the experiment. The comprehensive information about spectroscopic parameters of a molecule can be used for subsequent calculations of molecular spectra both linear and multiphoton. So these calculations can be used to simulate the spectra of interest and to predict the behavior of a molecule in an intense laser field.

In this section the results are presented concerned with the calculation of excited vibrational state spectra of spherical top molecules. The spherical top molecules are of great interest from the isotope selective dissociation point of view. The wide known example is $\mathrm{SF}_{6}$ molecule. Multiphoton dissociation of $\mathrm{SF}_{6}$ was investigated in a lot of laboratories. So it is possible to compare the results of calculations with experimental one.

The program package was developed to calculate the rotational-vibrational spectra of excited vibrational states of IR active modes of spherical top molecules $\mathrm{XY}_{4}\left(\mathrm{~T}_{\mathrm{d}}\right)$ and $\mathrm{XY}_{6}\left(\mathrm{O}_{\mathrm{h}}\right)$ and dipole moments of transitions between excited vibrational states. The Hamiltonian to fourth order of approximation for $v_{3}$ mode is given by the following formula ${ }^{22,23}$ :

$$
\begin{aligned}
\hat{\mathrm{H}}= & v_{3} \cdot \mathrm{v}+\mathrm{B} \cdot \mathrm{J} \cdot(\mathrm{J}+1)+(\mathrm{B} \zeta) \cdot[\mathrm{R} \cdot(\mathrm{R}+1)-\mathrm{J} \cdot(\mathrm{J}+1)-1 \cdot(1+1)] \\
& +\mathrm{X}_{33} \cdot \mathrm{v} \cdot(\mathrm{v}-1)+\mathrm{G}_{33} \cdot[1 \cdot(1+1)-2 \cdot \mathrm{v}]+\mathrm{T}_{33} \cdot \sqrt{120} \cdot \hat{\mathrm{T}}^{404} \\
& +\mathrm{D}_{\mathrm{s}} \cdot[\mathrm{J} \cdot(\mathrm{J}+1)]^{2}+\mathrm{Z}_{3 \mathrm{~s}} \cdot \sqrt{5} \cdot \hat{\mathrm{T}}^{220} \\
& +\sqrt{120}\left(\mathrm{Z}_{3 \mathrm{t}} \hat{\mathrm{T}}^{224}+\mathrm{D}_{\mathrm{t}} \hat{\mathrm{T}}^{044}+\mathrm{F}_{3 \mathrm{t}} \hat{\mathrm{T}}^{134}+\mathrm{N}_{333 \mathrm{t}} \hat{\mathrm{T}}^{313}\right)
\end{aligned}
$$

where $\mathrm{v}$ is vibrational quantum number, $\mathrm{J}$ is quantum number of a total angular 
moment, 1 is quantum number of a vibrational angular moment, $\mathbf{R}$ is quantum number of a vector formed by $\vec{J}$ and $\overrightarrow{\mathbf{I}}$ according to subtraction rule $\vec{R}=\vec{J}-\vec{I}$; rotational parameters of the Hamiltonian are defined by relations: $\mathrm{B}=\mathrm{B}_{0}+\mathrm{v} \cdot \mathrm{Y}_{3}$ is rotational constant of vibrational state, $(B \zeta)=(B \zeta)_{0}-M_{33} \cdot v-F_{3 s} \cdot(J+1) / 2$ is Coriolis parameter for certain vibrational-rotational state. The notation of tensor operators $\hat{\mathrm{T}}^{\mathrm{nmk}}$ is given in the form known from Reference 24 . In this notation the operator of dipole interaction between field and the dipole moment of vibrational mode is

$$
\vec{E} \cdot \vec{d}=-\sqrt{3} d_{01} E \cdot \hat{T}^{110}
$$

where $d_{01}$ is dipole moment of a fundamental transition of a mode; $E$ is amplitude of light field; the scalar product results in tensor operator $\hat{\mathrm{T}}^{110}$. The Hamiltonian (9) do not take into account the sextic intramode anharmonicity term which contribution is of fourth order and has nonzero matrix elements in vibrational states $v \geqslant 3$. The corresponding term in the Hamiltonian of IR active modes of spherical top molecules was analyzed in Reference 25:

$$
\begin{aligned}
\hat{\mathrm{H}}_{6}= & \delta \mathrm{X}_{33} \mathrm{v}(\mathrm{v}-1)(\mathrm{v}-2)+\delta \mathrm{G}_{33}[1(1+1)-2 \mathrm{v}](\mathrm{v}-2) \\
& +\sqrt{120} \delta \mathrm{T}_{33} \hat{\mathrm{T}}^{404}+\mathrm{T}_{433} \hat{\mathrm{P}}^{4}+\mathrm{T}_{633} \hat{\mathrm{T}}^{66}
\end{aligned}
$$

where five constants of sextic anharmonicity are defined and two tensor operators $\hat{\mathrm{P}}^{4}$ and $\hat{\mathrm{T}}^{66}$ introduced in Reference 25 are used. The sextic anharmonicity term was taken into account when calculating the excited state spectra.

Shown in Figure 11 are the results of calculations for $\mathrm{Q}$ branches of transitions $1 F_{1 \mathrm{u}}-2 \mathrm{~A}_{1 \mathrm{~g}}$ and $1 \mathrm{~F}_{1 \mathrm{u}}-2 \mathrm{E}_{\mathrm{g}}$ in comparison with experimental spectra observed in Reference 10 . These plots demonstrate that both frequency position and intensity of lines in calculated spectra are in good agreement with experimental one. The spectroscopic parameters known from the spectroscopic study of fundamental transition of $v_{3}$ of $\mathrm{SF}_{6}{ }^{26}$ were used in the calculations. Constants $\mathrm{X}_{33}, \mathrm{G}_{33}, \mathrm{~T}_{33}$ were fitted to reach an agreement between experimental and calculated spectra. These constants were found to be:

$$
\mathrm{X}_{33}=-1.7468(5), \quad \mathrm{G}_{33}=0.9262(2), \quad \mathrm{T}_{33}=-0.2487(1) \mathrm{cm}^{-1} .
$$

This set of quartic anharmonicity constants differs from that found in Reference 9 where the spectrum of overtone $0-3 v_{3}$ was analyzed. The difference is due to sextic anharmonicity constants of $v_{3}$ mode which was not included in the fit in Reference 9. So the comparison of quartic anharmonicity constants found in this work with those found in $0-3 v_{3}$ analysis gives a set of sextic anharmonicity constants:

$$
\delta \mathrm{X}_{33}=4.2 \cdot 10^{-3}, \quad \delta \mathrm{G}_{33}=-7.2 \cdot 10^{-3}, \quad \delta \mathrm{T}_{33}=2.35 \cdot 10^{-3} \mathrm{~cm}^{-1}
$$

with zero values at $T_{433}$ and $T_{633}$. The values of these constants show that it necessary to include the sextic anharmonicity terms in the Hamiltonian of $v_{3}$ mode to calculate the spectra of excited states with $10^{-3} \mathrm{~cm}^{-1}$ accuracy. Indeed, the contribution of sextic anharmonicity to the energy of $v=3$ state has the order of magnitude $10^{-2} \mathrm{~cm}^{-1}$ and for $\mathrm{v}=6$ state the contribution is about $0.5 \mathrm{~cm}^{-1}$. 


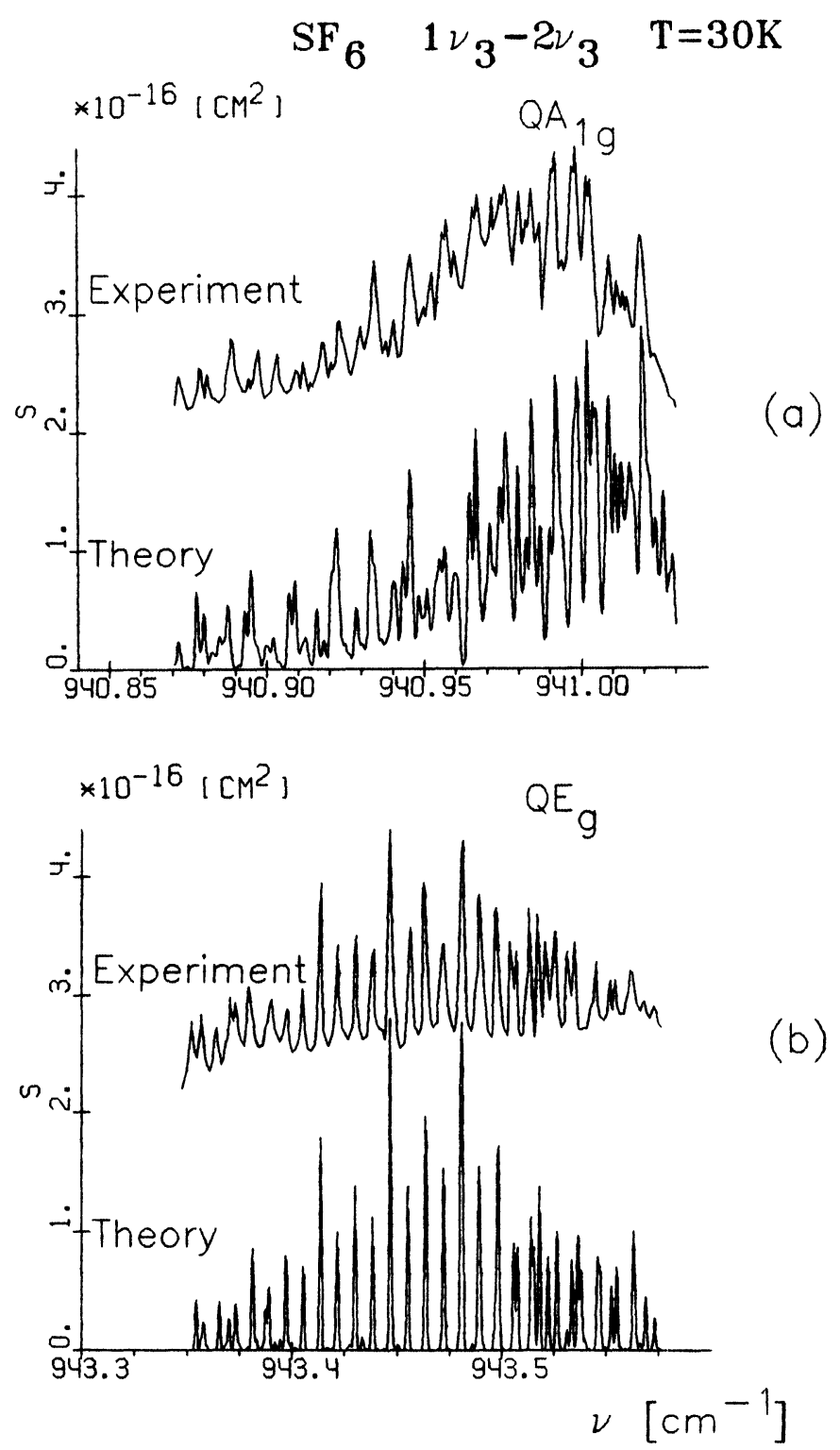

Figure 11 High resolution spectra of transitions $1 F_{1 u}-2 A_{1 g}$ (a) and $1 F_{1 u}-2 E_{g}$ (b). Upper curve-experimental data from Reference 10 , lower curve-calculated data.

The set of molecular spectroscopic constants found in the coarse of analysis of $1 v_{3}-2 v_{3}$ spectra was used to calculate rotational-vibrational lines of $2 v_{3}-3 v_{3}$ transition of $\mathrm{SF}_{6}$. Some of them were observed in Reference 10. Standard deviation between calculated and measured frequencies of the lines was found to be better than $10^{-3} \mathrm{~cm}^{-1}$. Thus, it demonstrates that this set of constants can be used to calculate 
the excited vibrational-rotational spectra of excited states of $v_{3}$ of $\mathrm{SF}_{6}$ with accuracy about $10^{-3} \mathrm{~cm}^{-1}$. However, there is a problem of breaking of the Hamiltonian of isolated mode (9) due to anharmonic coupling of $v_{3}$ with other vibrational states of molecule. It is this coupling that results in mode mixing and QC formation. So if the energy deposited in the molecule is more than boundary between DL region and QC the Hamiltonian (9) is not valid to describe the spectra of $v_{3}$ excited states. In $\mathrm{SF}_{6}$ this boundary was found to be near $\mathrm{v}=6$ level of $v_{3}$ mode. ${ }^{27}$ So the Hamiltonian (9) is likely valid to calculate the spectra up to $v=6$ of $v_{3}$.

Detailed agreement between experimental and calculated spectra makes it possible to restore full linear spectra of transitions between excited vibrational states. The survey linear spectra of successive one photon transitions in the $v_{3}$ ladder of $\mathrm{SF}_{6}$ $\mathrm{v}=0 \rightarrow \mathrm{v}=1, \mathrm{v}=1 \rightarrow \mathrm{v}=2, \mathrm{v}=2 \rightarrow \mathrm{v}=3$ and $\mathrm{v}=3 \rightarrow \mathrm{v}=4$ were calculated with the help of the developed program package. To smooth the detailed structure the line shape of individual rotational-vibrational transition was taken to be lorentzian with FWHM $0.05 \mathrm{~cm}^{-1}$. The spectra calculated at rotational temperature $\mathrm{T}=30 \mathrm{~K}$ are shown in Figure 12 and can be considered as computer restoration of full linear absorption spectra of transitions between excited vibrational states with deliberate reduced resolution. The spectra visualize that anharmonic and rotation-vibration coupling in the degenerate $v_{3}$ mode results in a great broadening of the spectra of transitions between excited vibrational states in contrast to the ordinary anharmonic shift of the spectrum due to anharmonicity in the case of one dimensional oscillator. This broadening makes it possible to overcome the detuning owing to anharmonicity in the coarse of resonant excitation of $v_{3}$ mode at fixed frequency of pumping radiation. Indeed, the absorption cross section of $\mathrm{v}=3 \rightarrow \mathrm{v}=4$ transition in the frequency region of fundamental transition is not zero and reaches $10^{-17} \mathrm{~cm}^{2}$ order of magnitude. The calculated spectra reveal also the narrow peaks due to overlapping of many rotational lines as in Q-branches and in other rotational branches of vibrational transitions. Q-branches of one photon transitions $3 \mathrm{~F}_{1 \mathrm{u}}-4 \mathrm{~A}_{1 \mathrm{~g}}$ and $3 \mathrm{~F}_{1 \mathrm{u}}-4 \mathrm{E}_{\mathrm{g}}$ near $931.8 \mathrm{~cm}^{-1}$ (energy gap between vibrational sublevels $\mathrm{A}_{1 \mathrm{~g}}$ and $\mathrm{E}_{\mathrm{g}}$ of $\mathrm{v}=4$ is very small) were observed in the three frequency dissociation spectrum (see above Figure $5 b$ ).

Next program package was created to simulate the excitation of molecules by intense IR radiation. The calculations of spectral dependencies of absorbed energy were based on a simple model. One and two photon transitions from ground vibrational state were taken into account. The subsequent excitation of molecules from second vibrational state of $v_{3}$ to excited states was described by successive one photon transitions with phenomenological absorption cross section given by relation (1). The pulse shape was taken to be rectangular.

The calculations were based on the following model. Interaction of laser pulse with discrete levels was described by Schrödinger equation:

$$
\mathrm{i} \cdot \hbar \cdot \psi=\mathrm{H} \cdot \psi+\overrightarrow{\mathscr{E}} \cdot \overrightarrow{\mathrm{d}} \cdot \cos (\omega \cdot \mathrm{t}) \cdot \psi
$$

The set of eigenvalues $\left\{\mathrm{E}_{\mathrm{v}, \mathrm{k}}\right\}$ of Hamiltonian $\mathrm{H}$ was calculated by first program package with $\mathrm{H}$ taken in form (9); $\mathrm{v}$ is vibrational quantum number, $\mathrm{E}_{\mathrm{v}, \mathrm{k}} \approx \mathrm{v} \cdot \hbar \cdot \omega$; $\mathrm{k}$ designates additional quantum numbers. Let $|\mathrm{v}, \mathrm{k}\rangle$ be eigenfunctions corresponding 

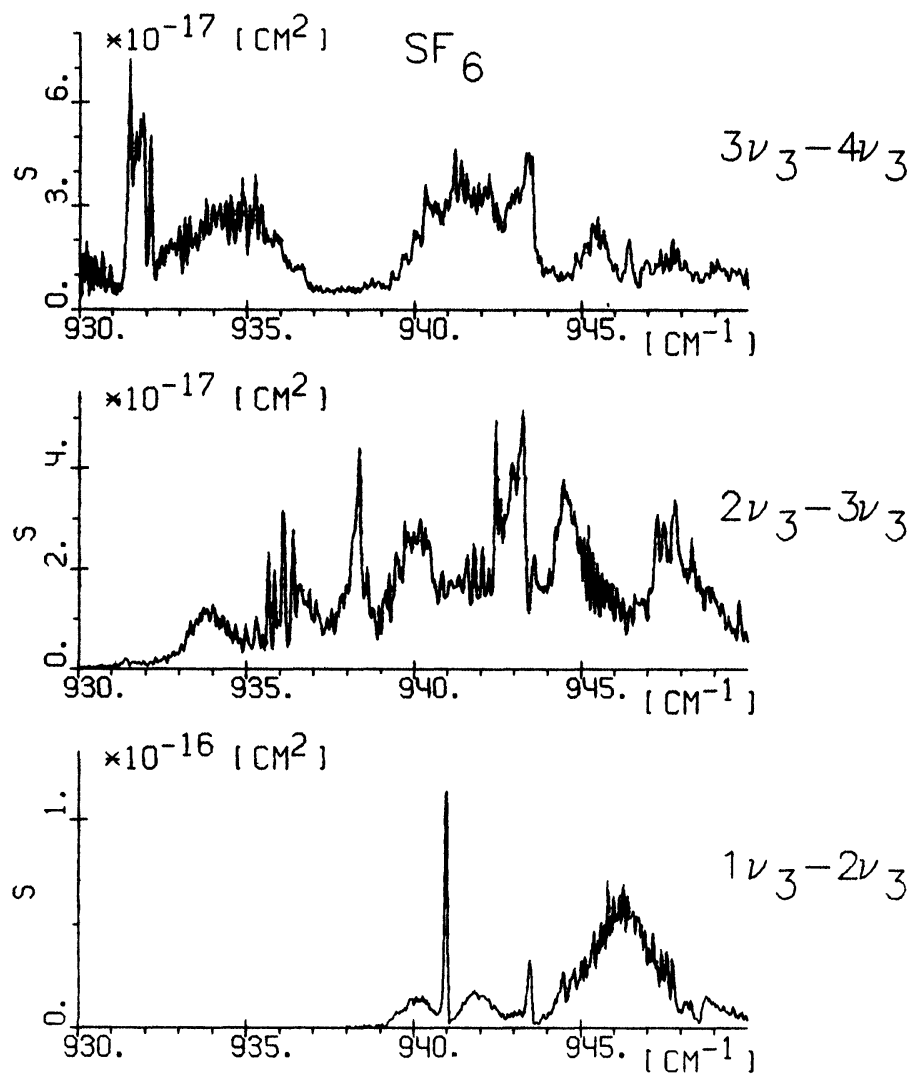

$1 v_{3}-2 v_{3}$
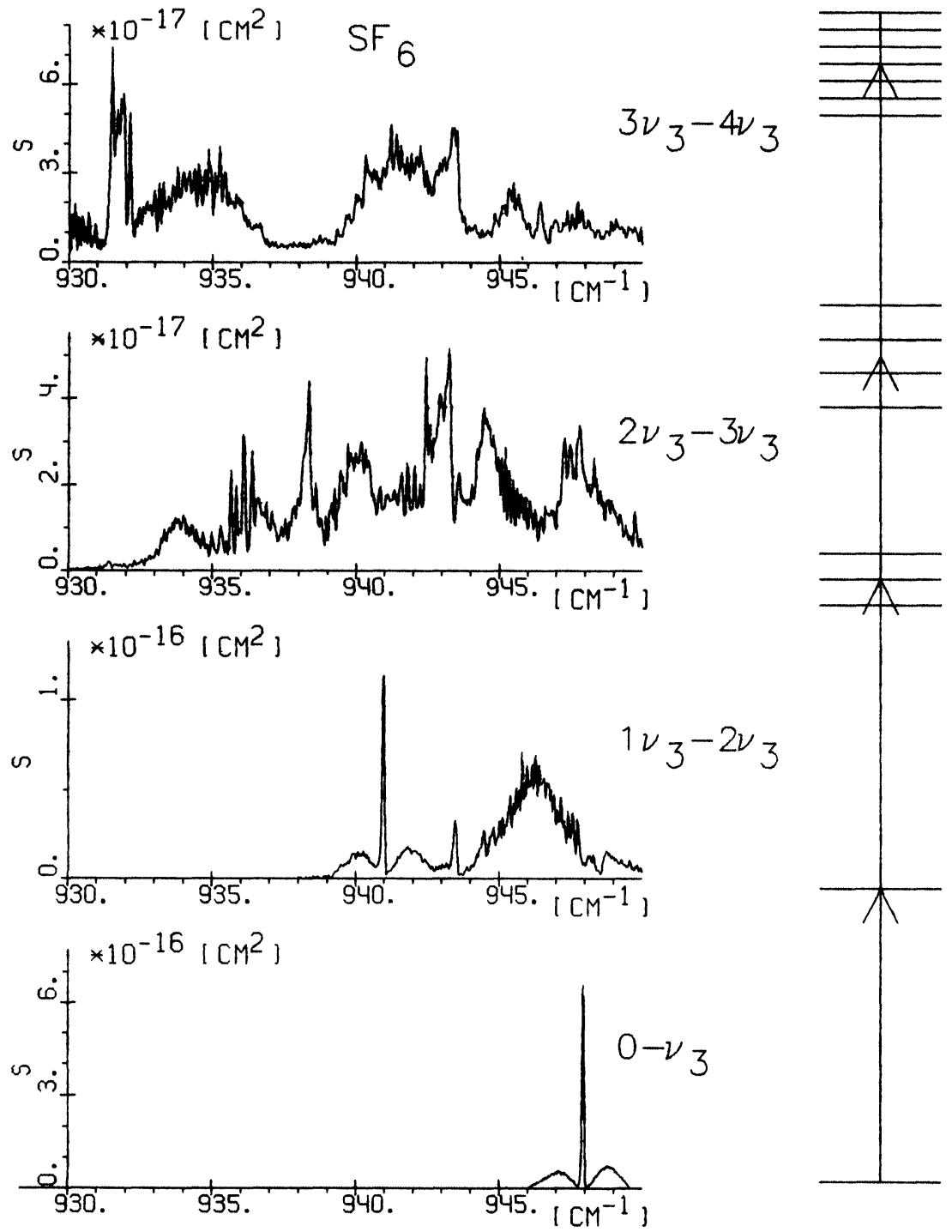

$\mathrm{A}_{2 \mathrm{u}}$

$\mathrm{F}_{2 \mathrm{u}}$

$\mathrm{F}_{1 \mathrm{u}}$

1u

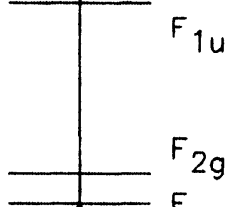

$2 \mathrm{~g}$

Figure 12 Survey spectra of successive one photon transitions in $v_{3}$ ladder of $\mathrm{SF}_{6}$.

to $\mathrm{E}_{\mathrm{v}, \mathrm{k}}$ then $\psi$ is represented by

$$
\psi=\sum_{\mathbf{v}, \mathbf{k}} \mathrm{a}_{\mathrm{vk}} \cdot|\mathrm{v}, \mathrm{k}\rangle
$$

From (12) we get equations for $a_{v, k}$

$$
\mathrm{i} \cdot \hbar \cdot \dot{\mathrm{a}}_{\mathrm{vk}}=\mathrm{E}_{\mathrm{v}, \mathrm{k}} \cdot \mathrm{a}_{\mathrm{vk}}+\mathscr{E} \cdot \mathrm{d}_{\mathrm{vk}, \mathrm{nm}} \cdot \mathrm{e}^{\mathrm{i} \omega \mathrm{t}} \cdot \mathrm{a}_{\mathrm{nm}} / 2+\mathscr{E} \cdot \mathrm{d}_{\mathrm{vk}, \mathrm{nm}} \cdot \mathrm{e}^{-\mathrm{i} \omega \mathrm{t}} \cdot \mathrm{a}_{\mathrm{nm}} / 2
$$


Transition to quasienergy representation

$$
\mathrm{a}_{\mathrm{vk}}=\sum_{1} \mathrm{~A}_{\mathrm{vk}, 1} \cdot \mathrm{e}^{\mathrm{i} 1 \omega \mathrm{t}}
$$

yields

$$
i \hbar A_{\mathrm{vk}, 1}=\left(\mathrm{E}_{\mathrm{v}, \mathrm{k}}-1 \cdot \hbar \cdot \omega\right) \mathrm{A}_{\mathrm{vk}, 1}+\mathscr{E} \cdot \mathrm{d}_{\mathrm{vk}, \mathrm{nm}}\left(\mathrm{A}_{\mathrm{nm}, 1-1}+\mathrm{A}_{\mathrm{nm}, 1+1}\right) / 2
$$

Eigenvalues of equations $(16)$ in the case of constant light field $(\mathscr{E}=$ const) are determined by

$$
\lambda \cdot \mathrm{A}_{\mathrm{vk}, 1}^{\lambda}=\left(\mathrm{E}_{\mathrm{v}, \mathrm{k}}-1 \cdot \hbar \cdot \omega\right) \mathrm{A}_{\mathrm{vk}, 1}^{\lambda}+\mathscr{E} \mathrm{d}_{\mathrm{vk}, \mathrm{nm}}\left(\mathrm{A}_{\mathrm{nm}, 1-1}^{\lambda}+\mathrm{A}_{\mathrm{nm}, 1+1}^{\lambda}\right) / 2
$$

Where $\lambda$ is quasienergy defined within the interval $-\hbar \cdot \omega / 2 \leqslant \lambda \leqslant \hbar \cdot \omega / 2$. General solution of (14) can be written as

$$
\psi=\mathrm{C}_{\lambda} \cdot \mathrm{A}_{\mathrm{vk}, \mathrm{l}}^{\lambda} \cdot \mathrm{e}^{\mathrm{i} \mathrm{l} \omega \mathrm{t}} \cdot \mathrm{e}^{-\mathrm{i} \lambda \mathrm{t} / \mathrm{\hbar}} \cdot|\mathrm{v}, \mathrm{k}\rangle
$$

where summation is over the repeated indexes. Coefficients $\mathrm{C}_{\lambda}$ determine the behavior of the quantum system. In the case, when $\mathscr{E}=0$ if $\mathrm{t}<0$ and $\mathscr{E}=$ const $\neq 0$ if $\mathrm{t}>0$, coefficients $\mathrm{C}_{\lambda}$ are determined by relations

$$
\mathrm{a}_{\mathrm{vk}}(\mathrm{t}=0) \sum_{\lambda} \mathrm{C}_{\lambda} \sum_{1} \mathrm{~A}_{\mathrm{vk}, 1}^{\lambda}
$$

The values $B_{\mathrm{vk}}^{\lambda}=\sum_{1} A_{\mathrm{vk}, 1}^{\lambda}$ are the elements of unitary matrix :

$$
\sum_{\lambda} \mathbf{B}_{\mathrm{nk}}^{\lambda} \cdot \mathbf{B}_{\mathrm{mj}}^{\lambda}=\delta_{\mathrm{nk}, \mathrm{mj}}, \quad \sum_{\mathbf{n k}} \mathbf{B}_{\mathrm{nk}}^{\lambda} \cdot \mathbf{B}_{\mathrm{nk}}^{\lambda^{\prime}}=\delta_{\lambda, \lambda^{\prime}}
$$

It follows for $\mathrm{C}_{\lambda}$ that

$$
\mathrm{C}_{\lambda}=\sum_{\mathbf{v k}} \mathrm{B}_{\mathbf{v k}}^{\lambda} \cdot \mathrm{a}_{\mathbf{v k}}(\mathrm{t}=0)
$$

The solution of above equations can be found explicitly if there is an exact resonance of exciting field with one of the transitions in the quantum system. Let a molecule is in the ground vibrational state with energy $\mathrm{E}_{\mathrm{o}, \mathrm{p}}$ (sublevel $\mathrm{p}$ ) and light field is in resonance with $\mathrm{n}$-photon transition

$$
\mathrm{E}_{\mathrm{n}, \mathrm{k}}-\mathrm{E}_{\mathbf{o}, \mathrm{p}}=\mathrm{n} \cdot \mathrm{h} \cdot \omega+\Delta_{\mathrm{nk}}
$$

where $\Delta_{\mathrm{nk}}$ is a small detuning. The solution of above equations can be found by means of perturbation theory technique. Compound matrix element $V_{n}$ of $n$-photon transition is determined by $\mathrm{n}$ order of perturbation theory as

$$
\mathrm{V}_{\mathrm{n}}=\left(\frac{\mathscr{E}}{2}\right)^{\mathrm{n}} \cdot \sum_{\mathrm{m}, \mathrm{i}} \prod_{\mathrm{v}=1}^{\mathrm{n}} \frac{\mathrm{d}_{\mathrm{vm},(\mathrm{v}-1) \mathrm{i}}}{\Delta_{(\mathrm{v}-1) \mathrm{i}}-\Delta_{\mathrm{vm}}}
$$

where summation is over all available intermediate levels, $d_{v m,(v-1) i}$ is dipole moment matrix element of transition $\mathrm{E}_{(\mathrm{v}-1), \mathrm{i}} \rightarrow \mathrm{E}_{\mathrm{v}, \mathrm{m}}$; detunings $\Delta_{\mathrm{v}, \mathrm{m}}$ are calculated in accordance with relation (22), by definition it is considered that in formula (23) $\Delta_{\mathrm{o}, \mathrm{p}}-\Delta_{1, \mathrm{i}}=1$.

Second order of perturbation theory determines the dynamic Stark shift of levels 
that results in changes in the detunings. As result the detuning of resonance of level $\mathrm{E}_{\mathrm{n}, \mathrm{k}}$ is found to be

$$
\Delta=\Delta_{\mathrm{nk}}+\sum_{\mathrm{m}} \frac{\left(\mathscr{E} \cdot \mathrm{d}_{\mathrm{nk},(\mathrm{n}-1) \mathrm{m}} / 2\right)^{2}}{\mathrm{E}_{\mathrm{n}, \mathrm{k}}-\mathrm{E}_{(\mathrm{n}-1), \mathrm{m}}-\hbar \omega}-\sum_{\mathrm{m}} \frac{\left(\mathscr{E} \cdot \mathrm{d}_{\mathrm{nk},(\mathrm{n}+1) \mathrm{m}} / 2\right)^{2}}{\mathrm{E}_{(\mathrm{n}+1), \mathrm{m}}-\mathrm{E}_{\mathrm{n}, \mathrm{k}}-\hbar \omega}+\sum_{\mathrm{m}} \frac{\left(\mathscr{E} \cdot \mathrm{d}_{\mathrm{op}, 1 \mathrm{~m}} / 2\right)^{2}}{\mathrm{E}_{1, \mathrm{~m}}-\mathrm{E}_{\mathrm{op}}-\hbar \omega}
$$

Summation must be done over all sublevels of the $\mathrm{v}=1, \mathrm{n}=1, \mathrm{n}+1$ states.

Only two quasienergy states or so called "dressed" states should be taken in account to compute the behavior of a system if there is only one resonance. In this case it is very simple to calculate the values of $A_{v k, 1}^{\lambda}$ and $C_{\lambda}$, then the value of any physical operator $\hat{\mathrm{P}}$ can be calculated by formula

$$
\langle\mathrm{P}\rangle=\mathrm{C}_{\lambda^{\prime}} \cdot \mathrm{A}_{\mathrm{v}^{\prime} \mathrm{k}^{\prime}, \mathrm{l}^{\prime}}^{\lambda^{\prime}} \cdot \mathrm{P}_{\mathrm{v}^{\prime} \mathrm{k}^{\prime}, \mathrm{vk}} \cdot \mathrm{C}_{\lambda} \cdot \mathrm{A}_{\mathrm{vk}, 1}^{\lambda} \cdot \mathrm{e}^{\mathrm{i}\left(\lambda^{\prime}-\lambda+\mathrm{l}^{\prime} \omega-1 \omega\right) t}
$$

The mean population of upper level of $n$-photon resonance can be calculated explicitly:

$$
\mathrm{N}_{\mathrm{nk}}=\frac{\mathrm{N}_{0}}{2 \cdot\left[1+\left(\Delta / \mathrm{V}_{\mathrm{n}}^{2}\right)\right]}
$$

where $\mathrm{N}_{0}$ is total population of resonant levels.

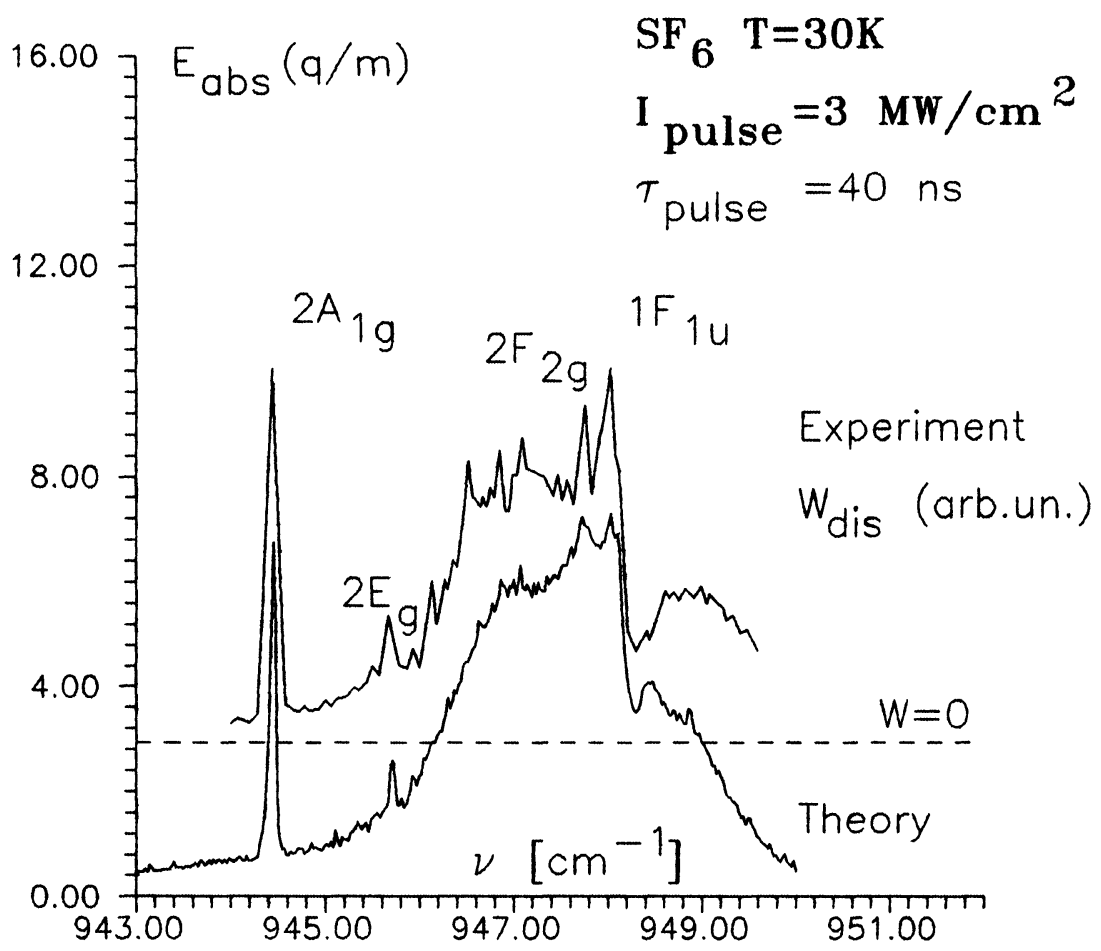

Figure 13 Calculated spectrum of absorbed energy and measured spectrum of dissociation of $\mathrm{SF}_{6}$. 
The program package included the program calculating all possible one and two photon resonances at given laser frequency, matrix elements of dipole moment of $v_{3}$ mode and population of upper level by formula (26). The subsequent excitation of molecules in the region upper than $\mathrm{v}=2$ was calculated in the frame of rate equations. This approach differs from those used in Reference 28 . We described excitation of $v \geqslant 3$ states with the help of rate equations and this simplification made it possible to utilize explicit formula to calculate dipole moment matrix elements while in Reference 28 dipole moment matrix elements were substituted by square averaged over M-projection. The latter made it possible to check polarization effects in the course of multiphoton excitation.

The simplifications of calculations were necessary because of limited power of computer employed (PC AT 386). The employment of more powerful computer makes it possible to calculate directly the contribution of three, four and more photon transitions in the excitation of vibrational ladder. But the results of calculations demonstrate that at laser pulse intensities less than $1 \mathrm{MW} / \mathrm{cm}^{2}$ this contribution cannot play a significant role.

The result of calculation with parameters (rotational temperature, pulse intensity, pulse duration, etc.) close to those in experiment is shown in Figure 13. Comparison with measured dissociation spectrum reveals a good agreement between experimental and calculated data. It points out that one and two photon transitions play the main

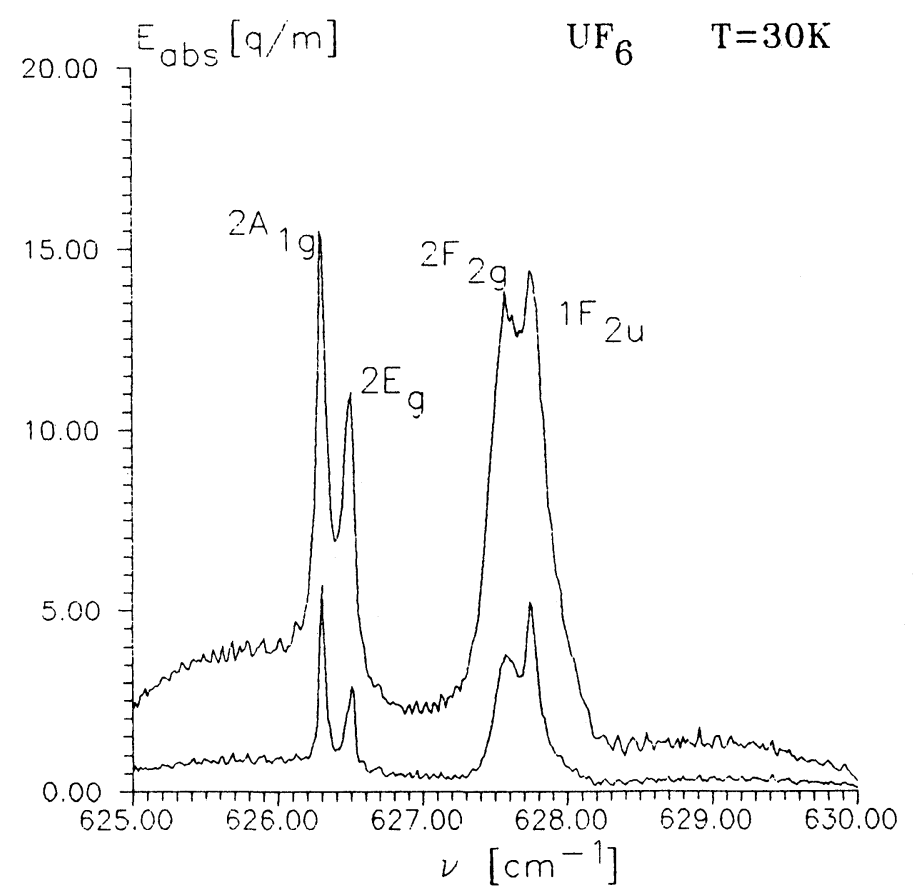

Figure 14 MPE spectra of $U_{6}$. Survey spectra calculated for laser pulse intensities $I=3 \mathrm{MW} / \mathrm{cm}^{2}$ (upper curve) and $\mathrm{I}=1 \mathrm{MW} / \mathrm{cm}^{2}$ (lower curve). Laser pulse duration was taken to be $100 \mathrm{~ns}$. 
role in the excitation of a molecule and determine the spectral dependence of DL excitation process at intensity of laser field about $1 \mathrm{MW} / \mathrm{cm}^{2}$ or less. The contribution of three, four and more photon transitions become significant if intensity is more than $1 \mathrm{MW} / \mathrm{cm}^{2}$. It should lead to smoothing of narrow peaks in spectral dependencies thus resulting in decreasing of selectivity of excitation. That is why it is multifrequency excitation that was used to decrease the intensity of laser field on the first stage of excitation with conserving of high efficiency of dissociation.

The program package was used to calculate MPE spectra of $\mathrm{UF}_{6}$ that is of practical interest. The spectroscopic constants of $\mathrm{UF}_{6}$ are known from linear spectroscopic measurements. ${ }^{29-31}$ The calculated MPE spectra are shown in Figure 14. The survey MPE spectra display the structure concerned with the two photon resonances. The peaks are narrow enough to reach an appreciable selectivity of excitation.

\section{Acknowledgements}

The authors would like to thank G. S. Baronov, N. V. Karlov, E. M. Khokhlov, V. S. Nersisjan, S. M. Nikiforov, A. L. Shtarkov and B. O. Zikrin for fruitful collaboration.

\section{References}

1. N. Isenor, V. Merchant, R. Hallsworth and M. Richardson. Can. J. Phys., 51, 1281 (1973).

2. R. V. Ambartzumyan, V. S. Letokhov, E. A. Ryabov and N. V. Chekalin. Pis'ma ZhETPh, 20, No. 9, 597 (1974) (in Russian).

3. V. N. Bagratashvili, V. S. Letokhov, A. A. Makarov and E. A. Ryabov. Multiple Photon Infrared Laser Photophysics and Photochemistry. Harwood Academic Publishers, p. 150 (1985).

4. Multiple Photon Excitation and Dissociation of Polyatomic Molecules B. Springer, p. 300 (1986).

5. S. S. Alimpiev, Yu. I. Bychkov, N. V. Karlov, E. K. Karlova, G. A. Mesyatz, Sh. Sh. Nabiev, S. M. Nikiforov, V. M. Orlovskii, V. V. Osipov, A. M. Prokhorov and E. M. Khokhlov. Sov. Tech. Phys. Lett., 5, 336 (1979).

6. S. S. Alimpiev, N. V. Karlov, G. A. Mesyats et al. JETP Lett., 30, No. 5, 259 (1979).

7. C. W. Patterson, F. Herlemont, M. Azizi and J. Lemaire. J. Mol. Spectr., 108, 31 (1984).

8. A. Linskens, S. te Lintel Heckert and J. Reuss. Infrared Phys., 32, 259 (1991).

9. C. W. Patterson, B. J. Krohn and A. S. Pine. J. Mol. Spectr., 88, 133 (1981).

10. S. S. Alimpiev et al. Opt. Spectrosc. (USSR), 67, 625 (1989).

11. S. S. Alimpiev, V. S. Nersisjan, S. M. Nikiforov and B. G. Sartakov. Quantum Electronics (USSR), 16, No. 4, 764 (1989) (in Russian).

12. Burger, H., Burszyk, K., Vichefeld, D. et al. Spectrochim. Acta., 35, No. 7, 875 (1979).

13. Burger, H., Burszyk, K., Bichefeld, D. et al. Spectrochim. Acta., 37, No. 8, 615 (1981).

14. Burger, H., Burszyk, K., Bichefeld, D. et al. Spectrochim. Acta., 38A, No. 6, 627 (1982).

15. S. S. Alimpiev, B. O. Zikrin, B. G. Sartakov and E. M. Khokhlov. Sov. Phys. JETP, 56, No. 5, 943 (1982).

16. P. N. Schatz and P. F. Horing. J. Chem. Phys., 21, 1516 (1953).

17. S. S. Alimpiev, N. V. Karlov, E. M. Khokhlov et al. J. Molec. Structure, 115, 229 (1984).

18. R. V. Ambartzumyan, V. S. Letokhov, G. N. Makarov and A. A. Puretzky. Opt. Commun., 25, 69 (1978).

19. Preprint LA-UR 78-858, Los Alamos, USA (1978).

20. D. G. Steel, R. C. Lind and J. F. Lam. Phys. Rev. A, 23, 2513 (1981).

21. V. N. Varakin and V. M. Gordienko. Pis'ma ZhETPh, 37, 188 (1983) (in Russian).

22. K. T. Hecht. J. Mol. Spectr., 5, 355 (1960).

23. J. Moret-Baily. J. Mol. Spectr., 15, 344 (1965).

24. A. G. Robiette, D. L. Gray and F. W. Birss. Mol. Phys., 32, No. 6, 1591 (1976).

25. B. G. Sartakov. In Infrared Laser Field Resonant Interaction with Polyatomic Molecules. Soviet Physics-Trudy IOFAN, Vol. 27, p. 3, Nauka (1990) (in Russian). 
26. B. Bobin, C. J. Borde, J. Borde and C. Breant. J. Mol. Spectr., 121, 91 (1987).

27. S. S. Alimpiev, A. A. Mokhnatyuk, S. M. Nikiforov, P. P. Pashinin, B. G. Sartakov, V. V. Smirnov and V. I. Fabelinski. CARS Spectroscopy of $S F_{6}$ Molecules Excited in a Strong IR Laser Field. Soviet Physics-Trudy IOFAN, Vol. 2, p. 136. Nauka (1986) (in Russian).

28. D. P. Hodgkinson, A. J. Taylor and A. G. Robiette. J. Phys. B. At. and Mol. Phys., 14, 1803 (1981).

29. J. P. Aldridge, E. G. Brock, H. Filip et al. J. Chem. Phys., 83, No. 1, 34 (1985).

30. M. Takami, T. Oyama, T. Watanabe, S. Namba and R. Nakane. Jpn. J. Appl. Phys., 23, L88 (1984).

31. C. W. Patterson. J. Chem. Phys., 83, No. 9, 4618 (1985). 